Jens Schlieter*

\title{
Religiöse Symbole im öffentlichen Raum: Symbolwirkung als kollektive Intentionalität einer Deutungsgemeinschaft
}

DOI 10.1515/zfr-2017-0009

Zusammenfassung: Wie im Aufsatz anhand von Fallbeispielen gezeigt wird, bietet die geläufige Bestimmung des religiösen Symbols, die von der Präsenz des Symbolisierten im Symbol und der Ähnlichkeit beider ausgeht, keine Hilfe bei der Analyse des Prozesses, wie ein Symbol im öffentlichen Raum religiöse Bedeutung erhält. Im Anschluss an John R. Searle wird anhand buddhistischer Deutungen ikonischer Symbole in Europa die Argumentation verfolgt, dass Symbole erst durch die kollektive Intentionalität von Deutungsgemeinschaften zu sozialen Tatsachen werden, deren Wirkmacht darin besteht, dass eine Gemeinschaft Interaktionen mit den ikonischen Symbolen unter normativen Erwartungen beobachtet. Gegen Theorien der „agency“ der Dinge wird vertreten, dass die Funktion der Symbole nur über sprachliche Zuschreibungen, genauer, über deklarative Sprechakte, sichergestellt wird. Aus deskriptiver Sicht muss zudem die Herstellungsabsicht berücksichtigt werden, mit der die Symbole geschaffen wurden, während religiöse Deutungen über diese oft hinweggehen. In Konflikten, die durch differente Deutungen über das Artefakt als religiöses Symbol oder als Kunstobjekt entstehen, kann nicht mit Verweis auf den „Transzendenzbezug“ des Symbols argumentiert werden. Die Herstellungsabsicht einbeziehend, sind somit die von religiöser Seite eingeforderten Achtungserweise vor dem Objekt vielmehr als Achtungserweise gegenüber der kollektiven Intentionalität der entsprechenden Deutungsgemeinschaft zu thematisieren.

Schlagwörter: Symbol, Symbolwirkung, kollektive Intentionalität, öffentlicher Raum, Buddhismus im Westen, Blasphemie

Abstract: The following contribution argues that the common definition of a religious iconic symbol, presupposing the similarity of the symbolized and the symbol, or a presence of the symbolized in the symbol, offers little help in analyzing the process in which a symbol assumes its status and role. The latter,

\footnotetext{
*Kontaktperson: Jens Schlieter, Universität Bern - Institut für Religionswissenschaft und Center for Global Studies; Lerchenweg 36, CH-3000 Bern 9, Schweiz, E-Mail:jens.schlieter@relwi.unibe.ch
} 
however, is of special relevance in those cases in which a "non-religious" symbol in the public sphere is endowed with religious significance. Applying the philosophy of John R. Searle, the contribution discusses cases of Buddhist iconic symbols in Europe. Symbols, it will be argued, become symbols through the collective intentionality of communities (religious or secular), and their efficacy, or "symbolic power", is nothing less (or no more) than the respective communities' normative assumptions on how interactions with the symbols shall take place. Against theories assuming an inherent agency of things and symbols, it will be argued that the functionality of symbols, or their "deontic power", can only be assured by declarative speech acts. Furthermore, from an etic perspective, the intention of the artist must be taken into consideration. Emic perspectives, in contrast, often define an iconic symbol without reference to its context of production. Conflicts, emerging from different interpretations of an artifact as either a religious symbol or a piece of art, cannot be solved by referring to a transcendence-reference inherent in the symbol itself. As a general consequence, the demand by religious actors of treating "symbols" as religiously significant and, in consequence, of normative expectations in regard to respectful forms of interaction, should not be discussed in relation to the iconic object, or symbol, in question. The relevant question is, rather, if - and how - the collective intentionality of the respective community shall be respected.

Keywords: icon, symbolic power, collective intentionality, public space, blasphemy 
Josef Simon (1930-2016), meinem Doktorvater, gewidmet

„Symbole sind Mystifikationen.“ „Das Äußre ist ein in Geheimniszustand erhobnes Innere. (Vielleicht auch umgekehrt.)“ Fragmente (22), Novalis (1772-1801)

„All die Vogelscheisse auf dem Steinbuddha. Recht so. Ich schwenke meine Arme wie Blumen im Wind.“ Ikkyū Sōjun (1394-1481)

\section{Das „religiöse Symbol“ und seine Wirkungen: Überblick des Arguments}

Eine Bestimmung, die von der Präsenz des Symbolisierten im Symbol und der Ähnlichkeit beider ausgeht, kommt bei genauerer Betrachtung des Prozesses, wie ein Symbol zu einem solchen gemacht wird, in unlösbare Konflikte. So wird mitunter gar davon gesprochen, dass ein religiöses Symbol eine „Ähnlichkeit“ zum Symbolisierten zeige, wobei das Symbolisierte auch nicht existieren könne. Symbole, so die unten verfolgte Argumentation, werden jedoch erst durch die kollektive Intentionalität von Deutungsgemeinschaften $\mathrm{zu}$ dem, was sie sind, nämlich: soziale Tatsachen. Letztere sind beobachter-relative Tatsachen und Institutionen, die ihre Funktion nur über sprachliche Zuschreibungen, also über deklarative Sprechakte, erhalten. Verpflichtungen wie etwa besondere Respektbekundungen diesen Symbolen gegenüber ergeben sich somit nur als Verpflichtungen in einer (ggf. religiösen) Deutungsgemeinschaft, die in kollektiver Intentionalität einem „, $\mathrm{x}^{\prime}$ den Status eines (ggf. religiösen) Symbols gibt und mit diesem Akt der Statuszuweisung zugleich solche Verpflichtungen institutionalisiert. Wie solche Prozesse zustande kommen, soll unten anhand von Fallbeispielen der buddhistischen Traditionen in Europa diskutiert werden, in denen Symbolwerdungsprozesse im öffentlichen Raum beobachtet werden können. Sie zeigen auf, dass aus deskriptiver Perspektive die Intentionen der Produzenten von ikonischen Objekten berücksichtigt werden müssen, während religiöse Akteure in emischer Sicht die den Produktionsprozess bestimmenden Intentionen oft unberücksichtigt lassen. Die politisch-rechtliche Frage, wie mit Objekten im öffentlichen Raum zu verfahren sei, die von bestimmten Akteuren oder Gemein- 
schaften zu religiösen Symbolen erklärt wurden, kann aus dieser Perspektive nicht beim „Symbol“ ansetzen, sondern bei der kollektiven Intentionalität der jeweiligen Deutungsgemeinschaften - religiöser wie säkularer.

\section{Religiöse Symbole im öffentlichen Raum - eine Problemanzeige}

Es wird in den letzten Jahren kaum möglich gewesen sein, sich der Debatte zu entziehen, ob der Vollschleier muslimischer Frauen ein religiöses Symbol darstelle oder nicht. In der Frage, ob die Verschleierung religiös geboten sei, kommen allerdings sowohl muslimische Frauen und religiöse Spezialisten des Islam wie auch Islamwissenschaftler $\mathrm{zu}$ durchaus unterschiedlichen Antworten. Bekanntlich ist in einigen europäischen Ländern (z.B. in Frankreich oder den Niederlanden) den muslimischen Trägerinnen das Anlegen eines Schleiers insgesamt oder in bestimmten Kontexten, in denen die gesellschaftliche Funktion eine weltanschauliche Neutralität gebiete, untersagt. ${ }^{1}$ Die juristische, politische und politikwissenschaftliche Debatte konnte hier auf Argumentationen zurückgreifen, die in dem zweiten strittigen Fall, der Anbringung von Kruzifixen in Schulräumen, bereits einige Jahre zuvor zu umfassenden Debatten und teilweise neuen staatlichen Regulierungen geführt hatte. Im Folgenden werde ich diese emotional hoch besetzten Beispiele nicht ausführlich diskutieren, da deren kontextuelle Rahmung und ein zumeist tief internalisiertes Vorverständnis den Blick auf die eigentliche Problematik in der Bestimmung religiöser Symbole und ihrer Wirkungen verstellen. Dennoch bin ich überzeugt, dass die unten geführte Diskussion auch für diese Symbole Implikationen mit sich bringt.

Relevant sind allerdings vor allem die Bestimmungen des religiösen Symbols und seiner Wirkungen, welche in juristischen und politischen Diskussionen begegnen. Nicht selten wird in diesen die „Wirkung“ eines Symbols (etwa in der Religionsphänomenologie oder den Entwürfen zur „symbolic power“) unmittelbar dem Symbol selbst zugeschrieben. In seiner Diskussion zur Rechtsprechung im Fall des Kreuzes - „das religiöse Hauptsymbol der christlichen Religionsrichtungen“, ${ }^{2}$ welches, wie das deutsche Bundesverfassungsgericht feststellte, ,appella-

1 Siehe vergleichend zur französischen Regelung Rudolf Steinberg, Kopftuch und Burka. Laizität, Toleranz und religiöse Homogenität in Deutschland und Frankreich (Baden-Baden: Nomos, 2015), 77-86.

2 Gerhard Czermak, Religions- und Weltanschauungsrecht. Eine Einführung (Berlin/Heidelberg: Springer Verlag, 2008), 148. 
tiven Charakter“3 habe - führt Gerhard Czermak aus: „Die religiöse Symbolwirkung kann nach dem Wesen von Symbolen auch nicht wegdefiniert werden. Wenn das Schulkreuz keinerlei Wirkung entfalten würde, gäbe es auch keinen verständlichen Grund, warum es angebracht werden sollte.”4 Aufgrund des „totalen Charakters des Symbolisierten“, so argumentiert Andreas Kley, ist den Symbolen selbst ein „Richtigkeits- und Herrschaftsanspruch“ inhärent, der, wenn die Symbole im öffentlichen Raum gezeigt werden, „zum Widerspruch herausfordern muss. [...] So wie das eine totale Gottesbild kein anderes neben sich dulden kann, so gilt das entsprechende notwendigerweise auch für die jeweiligen Symbole: Sie schließen sich aus, wenn sich die jeweiligen Anhänger nicht der Toleranz befleißigen." ${ }^{5}$ Kley verweist zwar auf die jeweiligen Deutungsgemeinschaften, bestimmt aber das Symbol und das diesem inhärente, das symbolisierte jeweilige „Heilige“, selbst als Agens. Ein religiöses Symbol, so heißt es in der Tradition der Religionsphänomenologie, stehe im „Themenbereich des Göttlich-Transzendenten“ für „das Höchste und Letzte, das Menschen denken und empfinden“6 können, d.h. „letztlich für Gottesvorstellungen“. Symbole sind „sinnlich wahrnehmbar“, überschreiten aber zugleich „die Grenze in die Welt der geistigen Vorstellungen und Ideen. Das, was eigentlich der menschlichen Anschauung entzogen ist, wird in Symbolen anschaulich und begreifbar vergegenwärtigt“. ${ }^{7}$

Im Hintergrund von Kleys Überlegungen wirkt die Vorstellung einer ,einheitlichen Symbolwirkung“ nach, die vor allem dadurch normiert wird, dass das Symbolisierte selbst den Anspruch auf Totalität zum Ausdruck bringt (und daher oft auch in toto abgelehnt werde). Lässt sich aber, so ist hier zu fragen, eine „Symbolwirkung“ oder „Symbolkraft“ von Symbolen ausmachen, die ausschließlich von der sinnlich wahrnehmbaren Präsenz eines „Symbols“ her konstruiert wird? Müssen nicht die vielfältigen Reaktionen anderer auf dieses Symbol, die sich keinesfalls nur in der Polarität von Zustimmung oder Ablehnung erschöpfen,

\section{Vgl.ebd.}

4 Ebd., 149.

5 Andreas Kley, „Kutten, Kopftücher, Kreuze und Minarette - religiöse Symbole im öffentlichen Raum," in Religion und Integration aus der Sicht des Rechts. Grundlagen - Problemfelder - Perspektiven, Hg. René Pahud de Mortanges (Zürich: Schulthess, 2010): 229-257,233.

6 Ebd.: 233.

7 Ebd.: 231. Wie genau dieses unsichtbare „Gegenstück“ konzeptuell gefasst werden soll, bleibt bei Kley jedoch unklar. Fraglos geht er allerdings von der Vorstellung eines quasi-ontologischen Passungsverhältnisses beider Seiten des Symbols aus: Die religiösen Symbole selbst, heißt es, „sind greif- und erfahrbar, wogegen das Verwiesene außerhalb menschlicher Sinneswahrnehmung liegt. Ein religiöses Symbol hat also gewissermaßen zwei Teile, einerseits den sinnlich wahrnehmbaren Gegenstand und [...] das dazugehörige geistige Gegenstück. Das Bild einer Scherbe, die genau auf ihr Gegenstück passt, trifft vollkommen zu“ (ebd.: 255). 
in die Bestimmung der „Symbolwirkung“ zentral einbezogen werden? Entscheidend ist hier die Mahnung von Karl-Heinz Ladeur, die „Komplexität der Wirkung kultureller Symbole“8 nicht aus dem Blick zu verlieren.

Freilich wird im Kontext juristischer Diskussionen oft darauf verwiesen, dass es hier weniger darum gehe, zu bestimmen, was ein religiöses Symbol sei, da im Prinzip alles zum religiösen Symbol werden könne. Die zentrale Frage sei daher, so Malcolm Evans, „not so much whether there is a religious symbol, or a matter which is of religious symbolic significance, which is at stake: rather, it is the context in which it occurs which matters most." Mit Referenz auf den Artikel 9 der Europäischen Menschenrechtskonvention (EMRK) ${ }^{10}$ richtet sich das Interesse der Juristinnen und Richter freilich weniger darauf, ob es sich um ein religiöses Symbol handele, sondern, ob es „Gläubigen“ (believers) erlaubt sein soll, es im öffentlichen Raum darzustellen. Die Frage ist somit, ob es sich bei dem sichtbaren Gegenstand oder der Bekleidungspraxis um eine Manifestation des Glaubens handele. Es gehe darum, so führt Evans im Kontext der Verschleierung und anderer Kleidung religiöser Gemeinschaften aus, dass diese überhaupt eine religiöse Einstellung manifestieren. Der zur Neutralität verpflichtete Staat könne nicht darüber entscheiden, welche Mittel - oder welche Symbole - für die Gläubigen orthopraktisch die richtigen seien, sondern nur, was ihre religiöse Verehrung in einer kontextuellen Situation gesellschaftlich bedeute. Es sei weniger relevant, was ein religiöses Symbol ist, sondern, "'what is understood to be religiously symbolic' in a given situation. This makes the matter more contextually rooted”. ${ }^{11}$ Die symbolische Sichtbarkeit wird somit auf „objects of religious veneration"12 umgelegt, wodurch sich die Argumentation des Bedürfnisses einer

8 Karl-Heinz Ladeur, Der Staat gegen die Gesellschaft: zur Verteidigung der Rationalität der ,Privatrechtsgesellschaft' (Mohr Siebeck: Tübingen, 2006), 165. Vgl. auch Rainer Forst, Toleranz im Konflikt - Geschichte, Gehalt und Gegenwart eines umstrittenen Begriffs (Frankfurt a.M.: Suhrkamp, 2003), 723.

9 Malcolm D. Evans, Manual on the Wearing of Religious Symbols in Public Areas (Council of Europe, Martinus Nijhoff Publishers, 2009), 61.

10 In Artikel 9, "Freedom of thought, conscience and religion”, wird festgehalten: "Everyone has the right to freedom of thought, conscience and religion; this right includes freedom to [...] manifest his religion or belief, in worship, teaching, practice and observance”. Diese Freiheit soll nur eingeschränkt werden „in the interests of public safety, for the protection of public order, health or morals, or for the protection of the rights and freedoms of others", so die European Convention on Human Rights (www.echr.coe.int/Documents/Convention_ENG.pdf [14.03.2017]; vgl. die Declaration on the Elimination of All Forms of Intolerance and of Discrimination Based on Religion or Belief, UN 1981, Art. 1, 1-3; http://www.un.org/documents/ga/res/36/a36r055.htm [14.03.2017]).

11 Evans, Manual, 68.

12 Ebd., 64. 
genaueren Bestimmung eines „religiösen Symbols“ entledigt. Dies führt letztlich dazu, dass der Versuch einer Klassifikation religiöser Symbole auf der Basis der Bestimmung „Objekt der Verehrung“ ganz aufgegeben wird. Zur „Idee eines Symbols“, so heißt es schlussendlich, gehöre es, dass sie solche Dinge enthalten könne, die, ,whilst not themselves objects of veneration, are representations of objects of veneration. An obvious example would be a crucifix or an icon. Of course, it is possible for representations of objects of veneration to become objects of veneration, thus making any classificatory approach impossible“.$^{13}$ Aus dem prinzipiell unabschließbaren und unvorhersehbaren Prozess, in welchem religiöse Gemeinschaften „Repräsentationen“ - die Frage wäre hier: auch nicht materielle, nicht sichtbare Repräsentationen? - vervielfältigen, die ihrerseits Objekte der Verehrung werden, folgt im juristischen Diskurs, dass der Fokus von der Bestimmung religiöser Symbole wiederum auf die Wirkung der Symbole verlagert wird: „Perhaps surprisingly, the real significance of something being a religious symbol lies in the response of others to that symbol“..$^{14}$

Zwar kann es, staatliche Neutralität sowie positive und negative Religionsfreiheit zusammengenommen, kein Recht geben, im öffentlichen Raum keinen religiösen Symbolen ausgesetzt zu sein. Dies führt Rainer Forst schlüssig in seiner philosophischen Studie zur Toleranz aus. ${ }^{15}$ Anders zu urteilen sei jedoch in jenen Fällen, in denen das Symbol für eine deutlich identifizierbare Praxis steht, die aus anderen, ethisch fundierten Gründen als unmoralisch erachtet wird und aus eben diesen verboten wurde. Zum anderen ist, wie Malcolm Evans an konkreten Rechtsprechungen verdeutlicht, jenes „sub-set of symbols which comprise objects of religious veneration" - mit Verweis auf den oben zitierten Artikel 9 der EMRK davor geschützt, zum Gegenstand ,provokativer Darstellungen“ (provocative portrayals) zu werden, wenn diese Darstellungen eine „malicious violation of the spirit of tolerance“16 zum Ausdruck bringen. Den Verletzungen der Gefühle der Gläubigen soll dabei ebenso Rechnung getragen werden wie auch dezidiert der Verletzung der Gefühle von Nicht-Gläubigen, wobei Streitfälle kontextuell und auf den Einzelfall bezogen erörtert werden. Mit der Referenz auf Gefühle und Emotionen wird die juristische Perspektive allerdings zur kontextabhängigen Güterabwägung. Es kann hier nur angedeutet werden, dass die Rechtsprechung regional und national entsprechend zu unterschiedlichen Ergebnissen kommt, je

\section{Ebd.}

14 Ebd., 66.

15 Vgl. Rainer Forst, Toleranz im Konflikt. Geschichte, Gehalt und Gegenwart eines umstrittenen Begriffs (Frankfurt a.M.: Suhrkamp, 2003), § 38.

16 Evans, Manual, 66, vgl. 17. 
nachdem, wie das Verhältnis dieser Güter zum säkularen Staat bzw. im öffentlichen Raum bestimmt wird.

Zusammenfassend kann festgehalten werden, dass in juristischen Äußerungen zum einen Teil Vorstellungen eines dem religiösen Symbol inhärenten Transzendenzbezugs präsent sind, welche auf die exklusive Totalisierung der Symbolwirkung hinauslaufen. In anderen Ausführungen stehen die Reaktionen anderer auf die (ggf. provokativ verfremdete) Darstellung von religiösen Symbolen im öffentlichen Raum im Mittelpunkt. In dieser Perspektive wird davon abgesehen, religiöse Symbole im Allgemeinen wie im Einzelfall in ihrer Referenz auf das Symbolisierte genauer zu bestimmen. Ausgangspunkt bildet die materielle Beliebigkeit der „Objekte der Verehrung“. Wenn allerdings die Praxis der Verehrung den Ausschlag gibt, stellt sich die Frage, wo die Grenze in Bezug auf mögliche „symbolische“ Objekte der Verehrung verläuft. Sind dies nur physische Objekte? Oder auch Namen (bzw. Worte und manifeste Wörter)? Spezifische Gesten und rituelle Handlungen? Oder können gar mentale Repräsentationen „Objekte der Verehrung“ sein? Das Symbol muss Eigenschaften besitzen, so kann mit Charles S. Peirce gefordert werden, die es erlauben, es von anderen Objekten zu unterscheiden. Wo genau, so kann am Fuß eines als heilig verehrten Berges stehend gefragt werden, verläuft die Grenze, hinter welcher der Berg als „object of veneration“ beginnt? Soll der Berg allein schon durch die ihm zuteil werdende Verehrung ein Symbol sein, so muss er hinreichend deutlich von dem, was er nicht ist, abgrenzbar sein. ${ }^{17}$

\section{Was sind „Religiöse Symbole“? Religionswissenschaftliche, zeichentheoretische und religionsästhetische Perspektiven}

In der Religionswissenschaft und anderen Wissenschaften - neben der Rechtswissenschaft sei hier nur auf die Religionssoziologie, die Theologie, die Sozialanthropologie verwiesen - ist der Begriff der „religiösen Symbole“ äußerst beliebt. Sowohl in der klassischen Religionsphänomenologie (van der Leeuw, Heiler, Eliade und andere) wie auch in der „Philosophie der symbolischen For-

17 Charles S. Peirce, Semiotische Schriften (Frankfurt a.M.: Suhrkamp, 2000), 1:188. Gefordert wird dies für alle Zeichen. 
men“ (Cassirer) und in der Theologie (z.B. Tillich) wurde vertreten, dass Menschen sich nur über Symbole das Heilige oder Göttliche vorstellen bzw. nur symbolisch von diesem reden könnten. ${ }^{18}$ In gegenläufiger Bewegung wurde Symbolen die Fähigkeit zugeschrieben, die Wirklichkeit des Heiligen zu enthüllen. Bei Eliade werden Symbole, die einen inneren Bezug zum Heiligen unterhalten und auf transhistorischen, archetypischen Symboliken aufbauen, zu einem prominenten Ort der Hierophanie. ${ }^{19}$ Religiöse Symbole garantieren als beobachter-unabhängige Gegebenheiten eine durch arbiträre Zeichen allein nicht sicherzustellende Bedeutungsfülle. Symbole, so heißt es weiter, verweisen intersubjektiv auf Transzendenz oder außeralltägliche Wirklichkeiten. Religiöse Gemeinschaften hingegen präsentieren sich kollektiv über emblematische Symbole oder vermögen es gar, nur durch Symbole sich selbst als Gemeinschaft zu repräsentieren. Religionswissenschaft wird dann zum „Symbolverstehen“ (Mensching). ${ }^{20}$ Auch wenn diese Beschreibung heutzutage in der Religionswissenschaft kaum mehr Zustimmung finden wird, muss dennoch gesagt werden, dass sie in der öffentlichen Wahrnehmung durchaus noch präsent ist. Erinnert sei an den Professor für „Religiöse Symbolologie“ („Religious Symbology“) an der Harvard Universität, Robert Langdon (seines Zeichens Protagonist der Bestseller-Romane von Dan Brown), der als derzeit bekanntester - wenn auch fiktionaler - Vertreter einer solchen „Religionswissenschaft“" gelten darf. ${ }^{21}$

Schlussendlich werden Religionen selbst mit Clifford Geertz oft als „Symbolsysteme“ definiert - ein Symbolsystem, das spezifische Wirkungen entfalten kann, nämlich „Stimmungen und Motivationen in den Menschen zu schaffen“, sowie ontologische und normative „Vorstellungen einer Seinsordnung“22 mit dem zur Deckung bringt, was jeweils als Wirklichkeit erfahren wird. Auch Ansätze der „material religion“ konzipieren die Symbolwirkung ausgehend von der physischen Präsenz des wahrnehmbaren „Symbols“ (oder icon) und erachten nicht selten - wenn auch als relationales Moment des Umgangs mit dem Symbol oder icon - dessen inneren Verweisungsbezug auf Transzendenz als zentral.

18 "Everything religion has to say about God", so Paul Tillich, "has a symbolic character", Systematic Theology (Chicago: University of Chicago Press, 1957; 2013), 2:9.

19 Vgl. Ulrich Berner, „Der Symbolbegriff in der Religionswissenschaft,” in Beiträge zu Symbol, Symbolbegriff und Symbolforschung, Hg. Manfred Lurker (Baden-Baden: Kröner, 1982): 17-28; hier $20-21$.

20 Vgl. ebd.: 19.

21 Vgl. vom Vf., „Wer hat Angst vor dem Dalai Lama? Victor und Victoria Trimondis Der Schatten des Dalai Lama (1999) als spiritualistische Verschwörungstheorie,“ Transformierte Buddhismen 1 (2008): 54-81, 56-59.

22 Clifford Geertz, „Religion als kulturelles System,” in Dichte Beschreibung, Ders. (Frankfurt a.M.: Suhrkamp, 1983): 44-95, 48. 
Birgit Meyer hingegen diskutiert Symbole bzw. icons im Zusammenhang mit den „sensational forms“, die autoritative mediale Formen des Zugangs zum Transzendenten darstellen, indem sie die Praktizierenden miteinander und für das Transzendente „engagieren“. ${ }^{23}$ Sicherlich ist der Rolle physischer Dinge, des Körpers und der Sinne als Konstituenten religiöser Praxis im öffentlichen Raum in religionswissenschaftlichen Ansätzen lange zu wenig Bedeutung beigemessen worden. ${ }^{24}$ Wenn aber nun Objekte, Dinge, Symbole oder icons jenseits von Sprache - hier vor allem: jenseits des Textuniversums der jeweiligen Deutungsgemeinschaften - und jenseits der Akte, mit denen sie zu intendierten Zwecken erschaffen wurden, als gleichsam selbsttätig kommunizierend beschrieben werden, wird eine magische Theorie der Bezugnahme reinstalliert, die nicht nur Theorieprobleme erzeugt, sondern zuletzt auch in ethische Dilemmata führt, wenn es um religiöse Symbole im öffentlichen Raum geht.

Wie aber werden diese Wirkungen des Symbols, von denen die Entwürfe sprechen, erreicht? Und wie lassen sich Symbole von Zeichen unterscheiden ${ }^{25}$ Ein genauerer Blick auf den in der Religionswissenschaft, der Sozialanthropologie sowie der Religionssoziologie zu findenden Begriffsgebrauch macht deutlich, dass nur selten genauer zwischen Symbol und Zeichen definitorisch unterschieden wird. Bereits Geertz nimmt, unter Rückgriff auf Susanne Langer, den Begriff des „Symbols“ in seiner weitestmöglichen Begriffsextension: Der Begriff Symbol, so Geertz, bezeichne „alle Gegenstände, Handlungen, Ereignisse, Eigenschaften oder Beziehungen, die Ausdrucksmittel einer Vorstellung sind, wobei diese Vorstellung die ,Bedeutung“ des Symbols ist“. ${ }^{26}$ Diese Definition von Symbol kommt allerdings schnell an ihre Grenzen, da sie die entscheidende Frage, wie die Beziehung des Symbols zum Symbolisierten hergestellt wird und beschrieben werden kann, im Dunklen lässt. So veranschaulicht Geertz seine Definition mit dem Beispiel der Zahl 6, die, wie er sagt, ein Symbol sei - egal, ob sie ,geschrieben, vorgestellt“, oder in einer beliebigen Einheit von sechs einzelnen Objekten

23 Vgl. Birgit Meyer, "From Imagined Communities to Aesthetic Formations: Religious Mediations, Sensational Forms, and Styles of Binding," in Aesthetic Formations. Media, Religion, and the Senses, Hg. Dies. (New York: Palgrave 2009): 1-28, 13, sowie insgesamt Religion, Media and the Public Sphere, Hg. Dies. und Annelies Moors (Bloomington: Indiana University Press, 2006).

24 Vgl. die berechtigte Kritik von Kim Knott, Birgit Meyer und Volkhard Krech, „Iconic Religion in Urban Space, “ in Material Religion 12/2 (2016): 123-136, 128.

25 Der in der Literatur- und Kunstwissenschaft geläufige Gebrauch des Begriffs „Symbol“ und seine Ableitungen (z.B. symbolische gegenüber wörtlicher Textauslegung, „Symbolismus“ usw.) kann im Weiteren nicht diskutiert werden, auch wenn offenkundig Querverbindungen zur Begrifflichkeit des „religiösen Symbols“ vorliegen.

26 Geertz, „Religion“: 49. 
präsentiert würde. Symbole sind also „Ausdrucksmittel“ bzw. „fassbare Formen von Vorstellungen“ wie auch die Vorstellungen selber, wodurch sich das Bezugsproblem zwar nicht erübrigt, aber gut platonisch in die Welt der Ideen verlagert wird. ${ }^{27}$ Irritierend ist allerdings, dass Geertz unmittelbar anschließend „Symbole“ als „aus der Erfahrung abgeleitete, in wahrnehmbare Formen geronnene Abstraktionen, konkrete Verkörperungen von Ideen, Verhaltensweisen, Meinungen, Sehnsüchten und Glaubensanschauungen" ${ }^{28}$ bestimmt. So sei es also möglich, anhand empirisch fassbarer Symbole, die in der sozialen Welt beobachtbar seien, wiederum die Vorstellungen zu untersuchen, deren Ausdruck sie sind. Bestimmungen dieser Art gehen von der Vorstellung einer magischen Bezugnahme von empirisch wahrnehmbaren Symbolen auf Vorstellungen - wenn nicht einer Identität beider - aus. Sie mögen dem emischen Diskurs über religiösen Symbole und Vorstellungen angemessen sein, nicht aber der theoretischen Reflexion auf diesen. Auch Symbole und ihre verschiedenen Bedeutungsgehalte müssen interpretiert und gedeutet werden. Ein Symbol kann daher auch missverstanden oder gar grundsätzlich nicht als Symbol erkannt werden. ${ }^{29}$

Geht es um eine präzise Definition, was „religiöse Symbole“ sind, gehört Unschärfe allerdings oft zum Programm. Ein besonderes Problem des in der Religionswissenschaft, aber auch der Wissenssoziologie geläufigen Begriffsgebrauchs wird manifest, wenn religiöse Symbole und Symbolsysteme unmittelbar mit Sprache und Kommunikation in Zusammenhang - wenn nicht gar: zur Deckung - gebracht werden..$^{30}$ Um hierfür nur ein Beispiel anzuführen: Burkhard

27 Vgl. ebd. Hier ist an Hans Blumenbergs Beobachtung (im Kontext des platonischen Höhlengleichnisses) zu erinnern: „Das Symbol verschließt sich gegen seine Herkunft, auch und gerade gegen die als Abbild. Es heraldisiert sich bis zur formalen Unkenntlichkeit, um ganz seiner Verweisung zu dienen: Verweisung auf das, was es selbst nicht ist, nicht sein darf und nicht sein kann“, so Ders., Höhlenausgänge (Frankfurt a.M.: Suhrkamp 1989), 164. „Symbole“, heißt es, führen aus der Höhle (cf. ebd., 167, mit Bezug auf Cassirer); kurz: „Das Symbol ersetzt nicht die Theorie, wie es die Theorie später selbst mit seinem Gebrauch will, sondern es macht sie überflüssig“ (ebd., 168).

28 Geertz, „Religion”: 49, Hervorhebung vom Vf. Einmal aus der Erfahrung abgeleitet, ein anderes Mal historisch überliefert: Diese Ambivalenz kennzeichnet auch den Begriff der Kultur nach Geertz „ein historisch überliefertes System überkommener Vorstellungen, die sich in symbolischen Formen ausdrücken, ein System, mit dessen Hilfe die Menschen ihr Wissen vom Leben und ihre Einstellungen zum Leben mitteilen, erhalten und weiterentwickeln“ (ebd., 46). Vgl. Moritz Klenk, „Religionswissenschaft als systemische Kulturwissenschaft. Überlegungen zu einer Synthese von Systemtheorie und kulturwissenschaftlicher Religionsforschung," Bayreuther Beiträge zur Erforschung der Religiösen Gegenwartskultur 2 (2011): 1-60, 12.

29 Vgl. Josef Simon, „Symbol I. Philosophisch,” TRE 32 (2001): 479-481.

30 Religion ist „immer auch eine symbolisch kommunizierte Wirklichkeit“, so unlängst Hubert Knoblauch, Die kommunikative Konstruktion der Wirklichkeit (Wiesbaden: Springer, 2017), 182, 
Gladigow bestimmt „Religion“ aus kulturwissenschaftlicher Perspektive programmatisch als „Kommunikations-, Deutungs- und Symbolsystem“ und „Religionen“ als „einen besonderen Typ eines kulturspezifischen Deutungs- und Symbolsystems [...], d.h. als Kommunikationssysteme mit einem bestimmten Zeichenvorrat und einer Reihe angebbarer Funktionen“. ${ }^{31}$ In der weiteren Ausführung wird ersichtlich, dass keine grundsätzliche definitorische Unterscheidung von „Zeichen“ (als „Wörter und Sätze“, „optische Zeichen“, „Bilder“, „Bewegungsabläu$\mathrm{fe}^{\text {“) }}$ und „Symbolen“ getroffen wird. Entsprechend ist nebeneinander von „Zeichensystem“ und von „Symbolsystem“ die Rede. ${ }^{32}$ So wird von „verschiedene[n] Klassen von ,Zeichen““33 gesprochen, die aber dieselben übergeordneten Funktionen ausüben und Bezugsrelationen aufweisen.

Allerdings muss Gladigow, der explizit auf Geertz aufbaut, zugestanden werden, dass die spezifisch kulturwissenschaftliche Unschärferelation des Gebrauchs der Begriffe des Zeichens und des Symbols weit verbreitet ist. Zudem erschweren die zahlreichen divergierenden Terminologien in Symbol- und Zeichentheorien im engeren (wie z.B. Karl Bühler, Charles S. Peirce) und weiteren Sinne (z.B. Sigmund Freud, Jacques Lacan, C.G. Jung, Claude Lévi-Strauss,

und es sind insbesondere „Kollektivsymbole“, die eine „enorm affektive Bedeutung“ haben, denn „sie repräsentieren das Ganze, Eine, Höchste - und wie immer die Kollektive legitimatorisch geordnet sind“ (ebd., 284). Da sich die symbolische Wirklichkeit der sozialen „entzieht“, werden Symbole als „besondere Zeichen“, nämlich als „Verweis“ erfahren (d.h., sie stellen Transzendenz im Sinne von Schütz her, vgl. ebd., 285). Knoblauch erachtet allerdings „Sprachzeichen“ als Untergruppe von Symbolen, wodurch, der Logik folgend, Sprachzeichen ebenfalls als „Verweise“ gelten müssten. Diese oft anzutreffende Ausweitung des Symbolbegriffs, die Sprachzeichen und ikonische Symbole in einem Atemzug verhandelt - „Symbole können in Stein gemeißelt sein“, treten aber auch als „kommunikative Formen [...] in Erscheinung. Es kann sich um Theater, um Bühnen oder um Bilder handeln, um Witze, Predigten“ (ebd., 286) -, verbaut die Möglichkeit, Prozesse der Bedeutungserzeugung von religiösen Symbolen genauer zu analysieren, denn deren isomorphe Passung und mögliche Ersetzung mit „symbolischer Sprache“ ist immer schon vorausgesetzt.

31 Burkhard Gladigow, „Gegenstände und wissenschaftlicher Kontext von Religionswissenschaft," in Handbuch religionswissenschaftlicher Grundbegriffe, Bd. 1, Hg. Hubert Cancik, Burkhard Gladigow, und Karl-Heinz Kohl (Stuttgart: Kohlhammer, 1988): 26-40, 32. „Zeichen“, heißt es dort, können „kognitive, emotionale, normative, soziale und kulturelle Prozesse auslösen, steuern und in Relationen zueinander setzen.“ Zu Gladigows Symbol-Begriff vgl. vom Vf., „,Religion, System, Symbol: Eine Diskussion der Voraussetzungen des „Drei-Ebenen-Modells“ von Religion," in Einheit und Differenz in der Religionswissenschaft, Hg. Karsten Lehmann und Ansgar Jödicke (Würzburg: Ergon Verlag, 2016): 75-95, 82-83.

32 So heißt es: „Unverkennbar ist die Tendenz, die Verbindlichkeit des Zeichensystems mit der Verbindlichkeit seiner Inhalte zu verknüpfen. Religiöse Symbolsysteme sind nicht einfach ,auszuwechseln““(Gladigow, „Gegenstände”: 34).

33 Ebd.: 35. 
Pierre Bourdieu, John R. Searle, Niklas Luhmann) ${ }^{34}$ die Diskussion. Die an Geertz anknüpfende Traditionslinie der Bestimmung des Symbols in der Sozialanthropologie, wie etwa bei Victor Turner, hat sich dem weiten SymbolVerständnis angeschlossen, so dass Turner - nicht von ungefähr Baudelaire zitierend - von einem „Wald von Symbolen“ spricht, in dem alles zum „multivokalen Symbol“ werden kann: natürliche Gegebenheiten, Handlungen, Vorstellungen, Menschen, Kräfte usw. Beliebig können diese je in neue symbolische Systeme eingegliedert werden. Den Symbolen selbst kommt dabei Wirkmächtigkeit bzw. „agency“ zu. Die Analyse von Ereignissen und Performanzen, so Turner, "involve symbols as agencies and foci of social mobilization, interaction, and styling of behavior". ${ }^{35}$

Turner definiert „Symbol“, indem er den „Signifikanten“, das sensorisch wahrnehmbare „Vehikel“ oder die „äußere Form“, und das „Signifizierte“ als Bedeutung, Sinn, Bezeichnetes, Denotat, Konnotiertes „of the symbol or sign“ unterscheidet. Das Symbol sei letztlich sowohl durch die Vielfalt seiner signata wie auch die Form seiner Bezeichnung vom Zeichen zu unterscheiden. Symbole, heißt es, zeigen "some kind of likeness between signifier and signified; in signs there need be no likeness". ${ }^{36}$

Auf das Argument einer Ähnlichkeitsbeziehung, welches Turner bei Ferdinand de Saussure entlehnt, ${ }^{37}$ wird unten einzugehen sein; es findet sich zudem in der Bestimmung des Symbols bei Peirce, bei dem es allerdings unter der Bezeichnung icon diskutiert wird. Peirce ist, wie erwähnt werden muss, überdies nicht unmittelbar auf die Diskussion religiöser Symbole eingegangen. Dennoch scheint es mir geboten, kurz auf seine Überlegungen zu verweisen, da seine Semiotik auch in evolutionären, theologischen und religionsästhetischen Entwürfen deut-

34 Vgl. den guten Überblick bei Eckard Rolf, Symboltheorien: Der Symbolbegriff im Theoriekontext (Berlin: De Gruyter, 2006).

35 Vgl. Victor Turner, „Symbolic Studies,” Annual Review of Anthropology 4 (1975): 145-161, 150151. Interessant ist, dass Turner allerdings sogleich dazu übergeht, diverse Beziehungen metaphorischer und metonymischer Art aufzuzeigen, die meines Erachtens nur auf der Ebene der Sprache Sinn ergeben.

36 Ebd.: 149.

37 Saussure definiert bekanntlich ein Zeichen als „arbiträr“, also in einem beliebigen und zufälligen Verhältnis zum Bezeichneten stehend. Ein Symbol hingegen ist ein „Bedeutungsträger“, bei dem zwischen der (sinnlichen) Form des Zeichens und dem, was es ausdrückt, noch ein Ähnlichkeitsbezug, ein „Rest einer natürlichen Verbindung“, bestehe. Bei Saussure heißt es in den Grundfragen der allgemeinen Sprachwissenschaft (Berlin/New York: De Gruyter, $\left.{ }^{3} 2001\right), 80$ : „Beim Symbol ist es nämlich wesentlich, dass es niemals ganz beliebig ist; es ist nicht inhaltlos, sondern bei ihm besteht bis zu einem gewissen Grade eine natürliche Beziehung zwischen Bezeichnung und Bezeichnetem.“ 
liche Spuren hinterlassen hat. ${ }^{38}$ Ein Zeichen - grundlegend nur in der Dreiecksrelation zwischen konkretem Zeichenvehikel, Objekt und Bedeutung erörterbar wird in erster Differenzierung entweder als „Ikon“, „Index“ oder „Symbol“ (symbol) bestimmt. Ein Index bleibt dabei als Fingerzeig auf sein präsentes Objekt verwiesen. Ein Symbol hingegen ist ein Zeichen, das sich entsprechend allgemeiner Vorstellungen, also durch Konvention, auf das Objekt bezieht. Es verlöre die Eigenschaft, die es zu einem Zeichen macht, wenn es keine Zeichenwirkung im (bzw. auf das) Bewusstsein des Interpreten gäbe. ${ }^{39}$ Von dieser Art, so Peirce, „ist jede sprachliche Äußerung, welche nur kraft dessen bedeutet, was sie bedeutet, weil sie so verstanden wird, daß sie Bedeutung besitzt.“40 Im Gegensatz dazu steht das icon in einer qualitativen Beziehung der Ähnlichkeit zum bezeichneten Objekt: Es sind Zeichen, „die sich auf ihre Objekte dank des unabhängigen Besitzes irgendeiner Eigenschaft dieser Objekte beziehen, wie z.B. die Figur eines Dreiecks, das in einer geometrischen Darstellung verwendet wird, um jedes beliebige Dreieck darzustellen, weil es drei geradlinige Seiten hat, die es genauso hätte, wenn es nicht als Zeichen aufgefaßt würde“. ${ }^{41}$ Offenbar gebraucht Peirce, sicherlich aber der Philosoph John R. Searle und andere, den Begriff symbol im Sinne des konventionell vereinbarten Zeichens, welches mit einer Interpretationsregel gedeutet wird. Das icon hingegen stehe in einer Ähnlichkeitsbeziehung zur Sache, auf die sie sich bezieht. ${ }^{42}$

Entscheidend ist nun die Auffassung von Peirce, dass, vereinfacht gesagt, eine Erklärung der „Bedeutung des Zeichens“ (also des „Interpretanten“) selbst wieder ein Zeichen erzeugt, das die Bedeutung des ersten Zeichens nicht erschöpft. Es verweist also wiederum auf ein weiteres Zeichen, welches zur Erläuterung der Bedeutung hinzugezogen werden müssen, usw. ad libitum. Wenn also etwas überhaupt als Zeichen verstanden wird, ist dessen Bedeutung

38 Vgl. z.B. Andrew Robinson, God and the World of Signs. Trinity, Evolution, and the Metaphysical Semiotics of C.S. Peirce (Leiden/Boston: Brill, 2010).

39 Peirce wird hier vereinfacht wiedergegeben. Seinerseits führt er den „Interpretanten“ ein, um dem Schluss auf ein dem semiotischen Prozess extern bleibendes, zeichendeutendes Bewusstsein entgegenzuwirken.

40 Peirce, Semiotische Schriften, 1:375.

41 Ebd., 1:266.

42 Um an den älteren (und insbesondere den deutschen) Sprachgebrauch anzuknüpfen, wird mit dem icon die Diskussion um das „Symbol“ fortgeführt, während das Peirce'sche symbol als „Zeichen“ zu übersetzen wäre. Wenn Peirce definiert, dass ein icon ein Zeichen sei, „das für sein Objekt steht, weil es als ein wahrgenommenes Ding eine Idee wachruft, die naturgemäß mit der Idee verbunden ist, die das Objekt hervorrufen würde“ (Semiotische Schriften, 1:204), so ist es genau diese Relation, die andernorts zur Grundlage der Definition des Symbols genommen wird. 
(oder, mit Luhmann: Sinn) nicht mehr negierbar. Die Explikation der Zeichen schreitet aber in einem Prozess der potentiell unendlichen Semiose fort, einer annäherungshalber unternommenen Verdeutlichung durch immer weitere Zeichen. ${ }^{43}$ Auch Peirce, so kann resümiert werden, geht von einem breiten Zeichenverständnis aus, das, unter zentralen Aspekten die Zeichen der Sprache, der Symbole, sowie der icons als einheitlich beschreibbar konzipiert. Zeichensysteme (inklusive der hier thematisierten emblematischen Symbole) werden, wie dies in anderen Entwürfen geschah, in toto vorausgesetzt. In der Suche nach einem Modell, in dem Prozesse der Erschaffung, Bedeutung und Verwendung ikonischer Symbole im Kontext religiöser Gemeinschaften so beschrieben werden können, dass die Frage, wie eine Deutungsgemeinschaft sich darauf einigt (oder auch nicht einigt), eine „Ähnlichkeit“ zwischen Symbol/icon und Symbolisiertem/object wahrzunehmen, hilft uns die Peirce'sche Großtheorie eines Meeres uferloser semiotischer Prozesse nur wenig weiter, auch wenn die Definition des icon als „Ähnlichkeit“ als „Goldstandard der Semiotik“ bezeichnet worden ist. ${ }^{44}$

Wie erwähnt, ist in der Religionsforschung zuletzt im Paradigma des „material turn“ und der Religionsästhetik die Arbeit am „religiösen Symbol“ bzw. „Ikon“ fortgeführt worden. Mit dem Ziel, der sinnlich vermittelten Materialität der Dinge in der religiösen Praxis neues Gewicht zu geben, wurde dabei von verschiedener Seite die Wirkmächtigkeit ikonischer Präsenz in den Mittelpunkt des Interesses gerückt - sei dies bei Hans Belting, Bruno Latour, David Morgan oder Birgit Meyer. Zwar wird zumeist zugestanden, dass die Institutionalisierung der Wirkmächtigkeit der Symbole sozial konstruiert sei. Dennoch werden diese Konstruktionsprozesse nicht weiter analysiert, sondern als Ausdruck einer Metaphysik der lebendigen Materialität genommen, die, mit den Gefühlen der religiösen Akteure authentifiziert, der „protestantischen“ Dematerialisierung und Weberschen Entzauberung der Welt thetisch entgegengesetzt wird.

Den Begriff iconic religion prägend, verwiesen zuletzt Kim Knott, Volkhard Krech und Birgit Meyer auf jene „symbolische Kraft“, die Objekte zu icons werden lasse. ${ }^{45}$ Die Frage nach der Beziehung der icons zum Dargestellten beantworten

43 Vgl. Josef Simon, Philosophie des Zeichens (Berlin/New York: De Gruyter, 1989), 232-237.

44 Vgl. Robert Yelle, „The Peircean Icon and the Study of Religion: A Brief Overview,” Material Religion 12/2 (2016): 241-243; 243; vgl. Ders., Semiotics of Religion: Signs of the Sacred in History (London: Bloomsbury, 2013).

45 Zitiert in Knott et al., „Iconic Religion”: 128: „Objects become icons when they have not only material force but also symbolic power. Actors have iconic consciousness when they experience material objects, not only understanding them cognitively or evaluating them morally, but also feeling their sensual, aesthetic force“ - so Jeffrey C. Alexander und Dominik Bartmański, „Intro- 
die Autoren wiederum mit der Ähnlichkeit beider: "The iconicity of religious icons consists of the likeness with and thus the presence of the signified attributed as religious and authorized respectively within a religious tradition." 46 Aus der Ähnlichkeit folgt - in der Bewegungsrichtung gut platonisch - die Präsenz des Symbolisierten im Symbol (icon), die allerdings anschließend sozial autorisiert wird. Weiter heißt es:

„Icons depict something which is not present (or even does not exist); they bring it to appearance (or even into existence) through bearing a resemblance with the depicted. However, likeness can only be realized via performative ascription. It only exists if a relation between an image and the depicted is called likeness and is authorized as such." 47

Wenn das ikonisch Symbolisierte auch nicht existieren kann, braucht es dann überhaupt das Kriterium „Ähnlichkeit“? ${ }^{48}$ Genügt es, die Kategorie „Ähnlichkeit“ beizubehalten, weil sie im emischen Diskurs ihren festen Platz hat und performative Handlungen sich (wiederum emisch) auf diese nicht weiter befragte Ähnlichkeit stützen? Und wenn Symbole bzw. icons selbst die Macht haben, das Symbolisierte, so es lediglich abwesend ist, präsent zu machen bzw. zu erzeugen: Wird dann dem bereits präsenten Symbolisierten in einem zweiten Schritt (jenem der performative ascription) der religiöse Sinn (i.e. die Ähnlichkeit) nur zugeschrieben und autorisiert? Meines Erachtens erscheint in dieser Konzeption der Zuschreibungsakt einmal als sekundär, da mit der „Ikonizität“ bereits ein prästabilisiertes Urbild-Abbild-Verhältnis vorausgesetzt wird. In der zweiten Formulierung erscheint die Zuschreibung als performative Erzeugung. Dieser Formulierung stimme ich gerne $\mathrm{zu}$ - die Ähnlichkeit des icon wird performativ erzeugt (inklusive der Sprechakte, die sagen, was hier „Ähnlichkeit“ heißen soll). Damit erübrigt sich allerdings der Bedarf für die theoretische Annahme einer Vorstellung des abwesenden oder gar nicht-existenten „Ähnlichen“, welches „präsent“

duction," in Iconic Power: Materiality and Meaning in Social Life, Hg. Dies. und Bernhard Giesen (New York: Palgrave Macmillan, 2012): 1-12, 1. Zwar ist auch hier wiederum von iconic power die Rede, welche den visuellen Objekten als actants mit eigener Willenskraft und Sozialleben (verwiesen wird auf Bruno Latour und Arjun Appadurai) zukommen, doch machen die Autoren immerhin deutlich, dass sie von (arcane) scripts ausgehen, in denen religiöse Bedeutung (wie z.B. Gebote) in die den Gläubigen sichtbaren Objekte „hineingeschrieben“ werden (vgl. ebd.: 1-3).

46 Ebd.: 130.

47 Ebd.

48 Hier ließe sich wohl mit Peirce erwidern, dass ein icon seinen Zeichen-Charakter behält, auch wenn es in concreto nicht als Zeichen fungiert (man denke hier an ein Verkehrszeichen, das eine Kurve anzeigt, aber abgebaut im Lager steht). Diese Antwort ergibt mithin nur Sinn, wenn das konkrete icon prinzipiell in einer Referenzrelation bekannt ist - vgl. Charles S. Peirce, Phänomen und Logik der Zeichen (Frankfurt a.M., 1983), 123-126. 
sei. ${ }^{49}$ Freilich betonen die Autoren, dass die „ikonische Qualität“ relational innerhalb einer „Mensch-Objekt Beziehung“ entsteht, doch liegt auch hier die Agency auf Seiten des ikonischen Objekts, mit welchem die Betrachtenden interagieren. Es ist das icon, welchem das Vermögen zukomme, „to enshrine and convey a sense of a special, sacrosanct presence to beholders whose acts and attitudes resonate with and reproduce this presence“. ${ }^{50}$ Schlussendlich wird also mit der Wirkmächtigkeit einer ikonischen Präsenz argumentiert, die sich im Auge des Betrachters entfaltet, weil sie bei diesem auf vorhandene Vorverständnisse („Resonanz") trifft.

\section{Religiöse Symbole, Zeichen und kollektive Intentionalität}

Um im Folgenden einen Anfang zu wagen, der möglichst unbelastet von den spezifischen Diskurskonstellationen ist, in denen das christliche Kruzifix und die Schleier muslimischer Frauen verhandelt werden, möchte ich mich im Weiteren auf Beispiele mit buddhistischen Akteuren beschränken. Eine weitere Eingrenzung, die sich methodisch motiviert, sei im Feld der „Symbole“ vorgenommen. Um mit handhabbaren Beispielen $\mathrm{zu}$ operieren, die sich analysieren lassen, möchte ich den Begriff (religiöses) „Symbol“ auf replizierbare, ${ }^{51}$ visuell und in Sonderfällen auch taktil wahrnehmbare Objekte eingrenzen, die im Folgenden „ikonische Symbole“ genannt werden. Diese haben „an sich“ dezidiert keine semantische Bedeutung, auch wenn jederzeit sprachliche, das Symbol erläutern-

49 Hier ließe sich einwenden, dass von der Ähnlichkeit mit nichtexistenten Dingen insofern sinnvoll gesprochen werden könne, als ja kulturell sedimentierte Vorstellungen in die Konstruktion des ikonischen Symbols mit einfliessen, die im nicht vollständig beliebigen Reservoir der Imaginationen der jeweiligen Tradition bereits vorhanden sind. Diesem Einwand ist jedoch zu entgegnen, dass es einmal einen ersten bedeutungsverleihenden Akt gegeben haben muss, auf dem diese soziale Praxis des „Ähnlichkeit-Sehens“ aufruht.

50 Knott et al., "Iconic Religion”: 129.

51 Die Replizierbarkeit, so könnte hier entgegengehalten werden, schließt unbegründeterweise aus, dass natürliche Objekte Symbol werden können. Das ist allerdings nicht so, denn es kann sowohl der „originale“ Bodhibaum, unter dem der Buddha der Tradition nach erwachte, Symbol für das Erwachen (Skt. bodhi) werden wie auch alle weiteren Replikate des Bodhibaums. Entscheidend für ikonische Symbole ist aber, dass Replikate angefertigt werden können. Ist dies nicht der Fall, dann bleibt unklar, wie Symbol und Symbolisiertes (bzw., in strukturalistischer Terminologie, Signifikant und Signifikat) überhaupt unterscheidbar sind bzw. das „Symbol“ nicht vielmehr die Sache ist. 
de Ausführungen möglich sind.${ }^{52} \mathrm{Ob}$ auch rituelle Handlungen, die ein identifizierbares und replizierbares Skript aufweisen, als Symbole bezeichnet werden können, sei hier dahingestellt. An den unten diskutierten Fallbeispielen wird freilich, wie erwähnt, deutlich werden, dass die weit verbreitete Definition der Sprache als Symbol bzw. symbolisch (von der Romantik über Cassirer bis hin zu Lacan, Bourdieu oder Berger und Luckmann) einen präziseren Begriffsgebrauch verunmöglicht. Auch ist Skepsis geboten, wenn Theorien der „symbolischen“ Bedeutung von Sprache verschiedenste Formen der, wie es heißt, nicht wörtlich zu verstehenden (nicht literalen) Rede - von Metaphern über Gleichnisse bis hin zu Allegorien - mit emblematischen Symbolen in eine Kategorie fassen. Dies führt zu zahlreichen Folgeproblemen wie der offenen Frage, wie die Semantik von nicht-sprachlichen Symbolen zu bestimmen sei. ${ }^{53}$ Die Antwort, die Bedeutung des Symbols könne ja durch einen „symbolisch“ verwendeten Ausdruck sprachlich wiedergegeben werden, führt hier nicht weiter, sondern perpetuiert lediglich das Problem.

Wenn hier nun religiöse Symbole als Zeichen behandelt werden, möchte ich nicht der oben beklagten Unschärfe Vorschub leisten, die beide Begriffe parallel verwendet. Die grundlegende Frage, unter welchen konkreten Voraussetzungen von religiösen Symbolen, die etwas anderes als Zeichen seien, gesprochen werden kann, wird am Schluss nochmals zu diskutieren sein.

Um die Probleme einer ontologischen Definition des „Symbols“ als nichtarbiträrem, transzendenz-bezogenem Zeichen einerseits und als Objekt der Verehrung andererseits zu diskutieren, adaptiere ich ein bei Hilary Putnam zu findendes Beispiel. Man stelle sich vor, dass eine Ameise im indischen Wüsten-

52 Vgl. die Argumentation von Hans H. Penner, „Why Religious Symbols Don’t Mean Anything,” in Religion im kulturellen Diskurs, Hg. Brigitte Luchesi und Kocku von Stuckrad (Berlin/New York: De Gruyter, 2004): 151-165, 158-159.

53 Vgl. dazu ebd.: 157-159. Ich folge Penner in der Argumentation, dass der technische Begriff „Bedeutung“ auf semantische Bedeutung eingegrenzt werden muss, die nur auf der Ebene von Sätzen bestimmbar ist. Sprache im gleichen Atemzug mit Symbolen zu nennen, ist mehr als unglücklich. Wenn - wie übrigens schon bei Aristoteles - „Sprachsymbole“ einbezogen werden, kann in der Tat alles, und überdies in jeder Dialogsituation, als Symbol aufgefasst werden. Ein beliebiger Satz könnte dann symbolische Dignität einfordern oder als blasphemischer Umgang mit „Sprachsymbolen“ anderer aufgefasst werden. Um diese und andere Folgerungen auszuschließen, sollen Symbole hier, wie gesagt, auf ikonische Symbole begrenzt werden. Dies ermöglicht, die Texte und Sprechakte, die entscheidend zur Dynamik der Konflikte von nunmehr „religiösen“ Symbolen im öffentlichen Raum beitragen, gesondert zu analysieren. Doch auch grundsätzlich gesehen wird ein übergreifendes Modell der Zeichen der Sprache nicht gerecht, die, auf semantischer Ebene, im Vergleich zu ikonischen Symbolen ein absolutes Mehr an Ausdrucksmöglichkeiten auch des „Unbegrifflichen“ birgt (z.B. Metaphern, Allegorien usw.). 
sand umherkriecht und dabei Linien im Sand zieht, in deren Folge eine Zeichnung entsteht, die deutlich als achtspeichiges Rad erkennbar ist. Durch einen hochgradig unwahrscheinlichen Zufall entspricht die Abbildung des Rades genau der geläufigen Ikonologie des buddhistischen Symbols des dharmacakra ( welches den „,achtfachen Pfad“ - seinerseits Quintessenz buddhistischer Praxis zum Ausdruck bringt. Kann gesagt werden, dass eine solche Zeichnung als Symbol etwas abbildet, bzw. auf etwas referiert? ${ }^{54}$ Putnam argumentiert, dass nicht von einem Abbild gesprochen werden kann, da die Ameise kein solches zeichnen wollte, und die Zeichnung daher in keiner Repräsentationsbeziehung zu einem Objekt, oder auch einer Vorstellung von einem Objekt, steht. Eine bloße Ähnlichkeit, und sei sie auch noch so deutlich, reicht hier nicht aus. Das Abbild kann erst Abbild sein, indem die Absicht des Verfertigenden darauf gerichtet ist, es als Abbild - mit einem referentiellen Bezug zu dem, was es darstellt - zu erschaffen, ${ }^{55}$ bzw., genau diesen Zusammenhang verstehend, ein Rezipient es überhaupt als Bild, Abbild, Symbol oder Zeichen auffasst. ${ }^{56}$ Es gibt hier also kein Symbol ,achtspeichiges Rad“ als objektive Tatsache. Das Verstehen des Symbols setzt voraus, dass zugleich die referentielle Absicht, in der es hervorgebracht wurde, mit verstanden wird. Ein ikonisches Symbol (wie auch ein Zeichen generell) ist nicht von Natur aus ein Symbol, sondern wird zu diesem, indem es ein durch den Interpreten verstandenes Zeichen ist - Missverständnisse in Hinblick auf die Intention seiner Verwendung (und nicht: seiner Bedeutung) eingeschlossen. ${ }^{57}$ Eine Bedeutung erhält das ikonische Symbol nur dann (und nur solange), wenn (und wie) es durch ein sprachlich artikuliertes, semantisches Korrelat ersetzt werden kann, welches eine Deutungsregel in Form einer Aussage enthält, und über die Zulässigkeit der Ersetzung in einer Sprachgemeinschaft weitgehend Einigkeit herrscht. ${ }^{58}$ Es kann also, mit anderen Worten, jederzeit die Frage nach der Bedeutung des Symbols (wie auch eines Zeichens) gestellt werden, aber durch das Antworten selbst wird der semantische Raum des Diskursiven betreten und zugleich die Ebene des emblematischen Symbols verlassen. Religionsgeschichtlich kann beobachtet werden, so meine These, dass Symbole oft aus einer metaphorischen Sprachverwendung hervorgehen, die erst in einer späteren Phase ikonisch umge-

54 Hilary Putnam, Vernunft, Wahrheit und Geschichte (Frankfurt a.M.: Suhrkamp, 1990), 15-16. 55 Interessanterweise bezeichnet Putnam die gegenteilige Auffassung als eine des „magischen Zusammenhangs“ (z.B. zwischen „Name“ und „Namensträger“), ebd., 17.

$56 \mathrm{Vgl}$. Simon, Philosophie des Zeichens, 45-46.

57 Vgl. ebd., 49.

58 Dies bezieht Position gegen die Idee der unendlichen Semiose, in der Zeichen immer nur durch andere Zeichen zu ersetzen sind. Das Problem ist wiederum, dass Sprache nur mit großen Verlusten an Theoriepotential als Zeichen (icon) bestimmbar ist. 
setzt wird. Der Symbolwerdungsprozess, der auch als Steigerung zur Selbstreferenz beschrieben werden kann, folgt in diesen Fällen auf einen bereits etablierten religiösen Diskurs über das, was dann semantisches Korrelat des Symbols werden wird. Solche Symbole entspringen nicht, wie in emischer Perspektive oft vertreten, einer Gleichursprünglichkeit von Symbol und Symbolisiertem, d.h. einer symbolischen Präsenz ab initio. ${ }^{59}$ Bevor das ikonische Symbol des Rades in gegenständlicher Form ungesetzt wurde - die buddhistische Tradition entfaltete sich bekanntlich über lange Zeit anikonisch -, begegnet das Rad bereits als Metapher in den Lehrreden des Buddha. Es versinnbildlicht als Metapher des ,achtfachen Pfads“ die Gesamtheit der buddhistischen Lehre sowie, in der Metaphorik des „Andrehens des Rads der Lehre“, die eigenständige Wirksamkeit der buddhistischen Befreiungslehre. Der Sinn des Letzteren ist klar: Die Lehre als „Rad“ ist gleichsam ein „Selbstläufer“. Die Metaphorik speist sich hier vor allem aus der Aufnahme realweltlicher Zusammenhänge, in denen Räder an Fahrzeugen Verwendung finden. ${ }^{60}$

Nun lässt sich das hypothetische Beispiel aber weiter ausbauen. Gesetzt, eine Gruppe tibetischer Buddhisten kommt an diesem Ort vorbei und verständigt sich kollektiv darüber, dass es sich um das Symbol des Rades handelt, obwohl sie die Ameisenspur als Ursache identifizieren. Den emischen Diskurs natürlich respektierend, der hier beispielsweise die Ameise als Bodhisattva verstehen könnte, welcher soeben das universelle Symbol des achtspeichigen Rades unter den Tieren bekannt machte, ${ }^{61}$ muss dennoch (aus deskriptiver Perspektive und mit dem Blick auf die Genese) auf die in Gänze fehlende Referentialität verwiesen werden. ${ }^{62}$ Dies schließt die ethisch relevante Frage, inwiefern das als Rad identifi-

59 So wurde beispielsweise erst die theologische Deutung des Todes Jesu am Kreuz entfaltet, bevor anschließend die Ablösung des Staurogramms durch das Kreuz und Kruzifix als zentralem Symbol des Christentums erfolgte.

60 Eine historische Analyse müsste in diesem Zusammenhang auch das Rad in seiner Herrschaftssymbolik (der cakravartin als dem Buddhismus zugeneigter „raddrehender Herrscher“) berücksichtigen, welche wahrscheinlich zu jener Zeit, spätestens aber in der Maurya-Dynastie, etabliert wurde.

61 Vgl. z.B. im Jātaka-Genre, oder die Erzählung von Avalokiteśvaras Unterweisung der Vögel in Gestalt eines Kuckucks (Tib. Bya chos rin chen 'phren ba), übersetzt von Edward Conze, The Buddha's Law Among the Birds (Delhi: Motilal Banarsidass, 1955). Generell darf wohl als eine bekannte religiöse Auffassung von Welt gelten, dass in ihr selbst in der Natur Symbole - als soteriologische Zeichen, vestigia usw. - „gelesen“ werden können.

62 Es sei betont, dass die hier getroffenen Bemerkungen zur fehlenden Referentialität nicht die Absicht verfolgen, einen naturalistischen Szientismus zu propagieren, der (a) ontologische Aussagen über eine Absenz des „Heiligen“ o.ä. für begründbar erachtet, oder der sich (b) über die religiöse Statuszuschreibung vollständig hinwegsetzt, sondern die Beschreibung der kollektiven Konstruktion von - in diesem Fall - religiöser Bedeutung zum Ziel haben. 
zierte und zum Gegenstand der Verehrung gewordene Bild eine kollektiv erzeugte Tatsache darstellt, natürlich nicht aus. Ein gedankenloses (oder gar intentionales) Hindurchlaufen könnte schon zu diesem Zeitpunkt zu einem Erweis von Missachtung werden. ${ }^{63}$ In dieser konkreten Kommunikationssituation findet ein relevanter Übergang statt - eine diskursive Verständigung, welche in einem Aushandlungsprozess die kollektive Intentionalität dieser Gruppe erzeugt, formt und so homogenisiert, dass die Individuen dieser Gruppe lernen, etwas als etwas zu sehen (im Sinne des „sehen als“ von Ludwig Wittgenstein ${ }^{64}$ ). Kollektive Intentionalität ist nun nach Searle nicht einfach eine Aufsummierung individueller Intentionalität. Vielmehr ist es ein eigener status quo, die „Wir-Intentionalität“, die etwa ermöglicht, dass eine Gruppe gemeinsame Ziele erreichen kann, ohne dass ununterbrochen die je individuelle Intentionalität auf diese Ziele ausgerichtet ist, oder auch von den je anderen Intentionalitäten gewusst werde. ${ }^{65}$ Searles Theorie sei hier mit Luhmann erweitert, in der Annahme, dass sich kollektive Intentionalität durch Verständigungen über Beobachtungen zweiter Ordnung bildet, nämlich, zu beobachten, wie andere das Bild als das wahrnehmen (lernen), als welches man selbst es wahrnimmt. ${ }^{66}$ Von entscheidender Bedeutung in diesem Prozess, kollektiv etwas als institutionelle Tatsache sehen zu lernen und mit seinen Verpflichtungen anzuerkennen, ist nun, so die Überlegung, weit weniger die eigene, sensomotorische Interaktion mit dem Gegenstand, sondern sprachliche Kommunikation. Denn erst in dieser wird ja das kollektive „sehen

63 In der deskriptiven Bestimmung von Ethik als Kommunikation von erwiesener Achtung bzw. Missachtung folge ich Luhmann, der seinerseits auf Talcott Parsons' Unterscheidung von esteem/ disrespect aufbaut. Vgl. Niklas Luhmann, „Paradigm lost. Über die ethische Reflexion der Moral,” in Paradigm lost. Über die ethische Reflexion der Moral, Hg. Ders. und Robert Spaemann (Frankfurt a.M.: Suhrkamp, 1990): 9-48, insbes. 17-18, sowie Ders. „Ethik als Reflexionstheorie der Moral,” in Gesellschaftsstruktur und Semantik, Ders. (Frankfurt a.M.: Suhrkamp, 1989): 3:358-448.

64 Vgl. die Diskussion der Überlegungen Wittgensteins bei Thomas Jantschek, „Bemerkungen zum Begriff des Sehen-als,” in Kritik des Sehens, Hg. Ralf Konersmann (Leipzig: Reclam, 1997): 299-319.

65 Vgl. Andrea Rota, „Religion as Social Reality. A Take on the Emic-Etic Debate in Light of John Searle's Philosophy of Society," Method and Theory in the Study of Religion 28/4 (2016): 421-444, 427.

66 In seiner Bestimmung des „symbiotischen Symbols“ führt der späte Luhmann aus, dass es sich „auf die körperlich mögliche Wahrnehmung“ beziehungsweise auf „die Möglichkeit des Wahrnehmens der Wahrnehmungen anderer“ stützt. Zwar stelle dies „keine letzte Entscheidungsinstanz“ für Wahrheit, wie in empiristischen Theorien behauptet, dar, „denn selbst wenn feststeht, daß wahrgenommen wurde, kann immer noch darüber gestritten werden, was wahrgenommen wurde [...]. Aber die Wahrnehmung des Wahrnehmens anderer sorgt für Irritation und kann nicht ohne weiteres ignoriert werden“, so Ders., Die Gesellschaft der Gesellschaft (Frankfurt a.M.: Suhrkamp, 2009), 379. 
als“ von einer sozialen Tatsache zu einer kollektiven Institution. Die bloße Beobachtung von individuellen Achtungserweisen - etwa: die Zeichnung im Sand nicht zu betreten - reicht nicht aus, denn dies könnte z.B. Ausdruck einer ästhetischen Wahrnehmung sein; wobei hier nicht bestritten werden soll, dass der kollektive körperliche Umgang (etwa in rituellen Handlungen) einen wichtigen Teil zur Internalisierung von Achtungserweisen beiträgt.

Um die zentrale Bedeutung von Sprache in der Erzeugung institutioneller Tatsachen durch kollektive Intentionalität herauszustreichen, kann auf das in diesem Punkt sehr aufschlussreiche psychologische Experiment „Princess Alice is watching you“ verwiesen werden. In dem Experiment wurden Kinder in einer Versuchsanordnung mit einer unsichtbaren Prinzessin Alice vertraut gemacht, die, wie die Versuchsdurchführenden sagten, sich auf einem (vermeintlich leeren) Stuhl im Raum befände. Mit der „Prinzessin“ im Raum allein gelassen, schummelten die Kinder in der Lösung einer schwierigen Aufgabe deutlich weniger als eine Vergleichsgruppe ohne die sprachlich evozierte Präsenz von Alice, da sich erstere offenbar beobachtet fühlten. ${ }^{67}$

Oben sind zur Beschreibung des Prozesses Überlegungen von John R. Searle eingeflossen, die noch etwas expliziter gemacht werden können. Mit Searle kann das Bild genau dann als „gesellschaftliche Tatsache“ erachtet werden, wenn in ihm eine kollektive Intentionalität enthalten ist. ${ }^{68}$ Die kollektive Einstellung, die zu der gesellschaftlichen Tatsache eingenommen wird, erzeugt diese überhaupt erst - zum Beispiel, indem eine Reihe von kleinen Steinen um ein Territorium in der symbolischen Funktion auftreten kann, „etwas jenseits seiner selbst anzuzeigen“ - eine Grenzlinie, die nur durch die kollektive Intentionalität existiert, mit der sie in der Deutungsgemeinschaft anerkannt wird. ${ }^{69}$ Es kommt, nebenbei gesagt, nicht von ungefähr, dass Searle hier ein Beispiel wählt, in welchem die institutionelle Tatsache durch einen kollektiven Akt der Anerkennung, wenn nicht gar des Respektes geschaffen wird. Der institutionellen Tatsache kommt dann eine Statusfunktion zu, die ihrerseits „deontische Macht“ ausübt, wie unten ausgeführt werden wird.

67 Vgl. J. Piazza et al. “'Princess Alice is Watching You': Children’s Belief in an Invisible Person Inhibits Cheating," Journal of Experimental Child Psychology 109/3 (2011): 311-320.

$68 \mathrm{Vgl}$. John R. Searle, Die Konstruktion der gesellschaftlichen Wirklichkeit. Zur Ontologie sozialer Tatsachen (Frankfurt a.M.: Suhrkamp 2011), 34-36.

69 Ebd., 48. In diesem Fall liegt allerdings, wie Searle andernorts zugibt, auch eine ,indexikalische“ Relation zum Bezeichneten vor, da sie ja auf der Grenze „liegen“ - vgl. John R. Searle, Geist, Sprache und Gesellschaft. Philosophie in der wirklichen Welt (Frankfurt a.M.: Suhrkamp, 2001), 184. 
Ein weiterer, entscheidender Schritt im Prozess der Erzeugung des emblematischen Radsymbols als institutioneller Tatsache wäre dann vollzogen, wenn nun Tibeter dieser Gruppe in ihrem Heimatort die Nachricht verbreiteten, dass an dem von ihnen indizierten Ort ein „Rad der Lehre“ im Sand zu sehen sei - und dies, ohne auf die Ameise zu verweisen -, und nun andere diesen Ort aufsuchen, um das Bild zu sehen und $\mathrm{zu}$ verehren (wobei in diesem Fall die Unbeständigkeit einer solchen Zeichnung der Eignung als Kultort natürlich deutliche Grenzen setzt). In diesem Fall ist das „Rad der Lehre“ semantisch mit einer Bedeutung angereichert, die in verbaler Artikulation an die Stelle des sichtbaren Emblems treten kann, weil in kollektiver Intentionalität davon ausgegangen wird, dass eben dies die Darstellungsabsicht der Zeichnung ist. Mit Searle gesprochen wäre dies ein Fall, in welchem performativen Äußerungen - genauer: deklarative Sprechakte, in welchen vermittels der Durchführung des Sprechaktes die institutionelle Tatsache geschaffen wird - entscheidend dazu beitragen, die institutionelle Tatsache zu erzeugen (wie etwa, indem eine dazu befugte Person spricht: „Ich erkläre Euch zu Mann und Frau“). ${ }^{70}$ Der Prozess der "Symbolwerdung“ ist vollendet, wenn die Zuweisung dieser Statusfunktion gelingt. ${ }^{71}$ Letzteres ist, wie gesagt, im Falle von institutionellen Tatsachen immer sprachabhängig. Ohne Menschen, welche in einer kollektiven „Wir-Intentionalität“ die Ansichten darüber, was hier vorliegt, teilen - etwas, was nur durch sprachliche Kommunikation in Erfahrung gebracht werden kann -, liegt hier nichts vor: keine Zeichnung, kein Symbol, keine ikonische Ähnlichkeit und kein Rad der Lehre. Während also in emischer Perspektive institutionelle Tatsachen geschaffen werden, die über die fehlende Referentialität hinweggehen können, müssen aus deskriptiver Perspektive die Deutungs- und Konstruktionsakte durch kollektive Gemeinschaften jenseits einer „Ähnlichkeit“ des als Symbol verstandenen „etwas“ beachtet werden dann jedenfalls, wenn ein Interesse daran besteht, die Genese eines Symbolwerdungsprozesses zu verstehen.

70 Vgl. ebd. 43-52. Ich gehe hier allerdings insofern über Searle hinaus, als die Intentionalität, mit der ein (religiöses) Symbol - zunächst überhaupt als erstes seiner Art, und dann als Replika geschaffen wird, Teil dessen ist, was durch die Deutungsgemeinschaft nachvollzogen wird, wobei, wie gesagt, im emischen Diskurs menschliche Intentionalität auch durch übernatürliche Intentionalität ersetzt werden kann.

71 Vgl. Rota, „Religion”: 428. 


\section{Vom „Mandala“ zum buddhistischen maṇạla $a^{72}$ oder: Wie ein religiöses Symbol entsteht}

Nun ließe sich gegen das obige Beispiel einwenden, dass es als Gedankenexperiment eines zudem hochgradig unwahrscheinlichen Falles zur Ontogenese religiöser Symbole im öffentlichen Raum nur wenig beitrage. Darum wird nunmehr im Weiteren auf aus der Lebenswelt entstammende Beispiele eingegangen. Deren erstes ist das Werk ,jetzt geht es um die Wurst“ des Berner Künstlers Bernhard Huwiler, welches anlässlich der Ausstellung „Reiz \& Risiko/Risk \& Allure“ im Haus für Kunst (Uri) angefertigt und am 7. Juli 2006 mit einer Performance eröffnet wurde. ${ }^{73}$

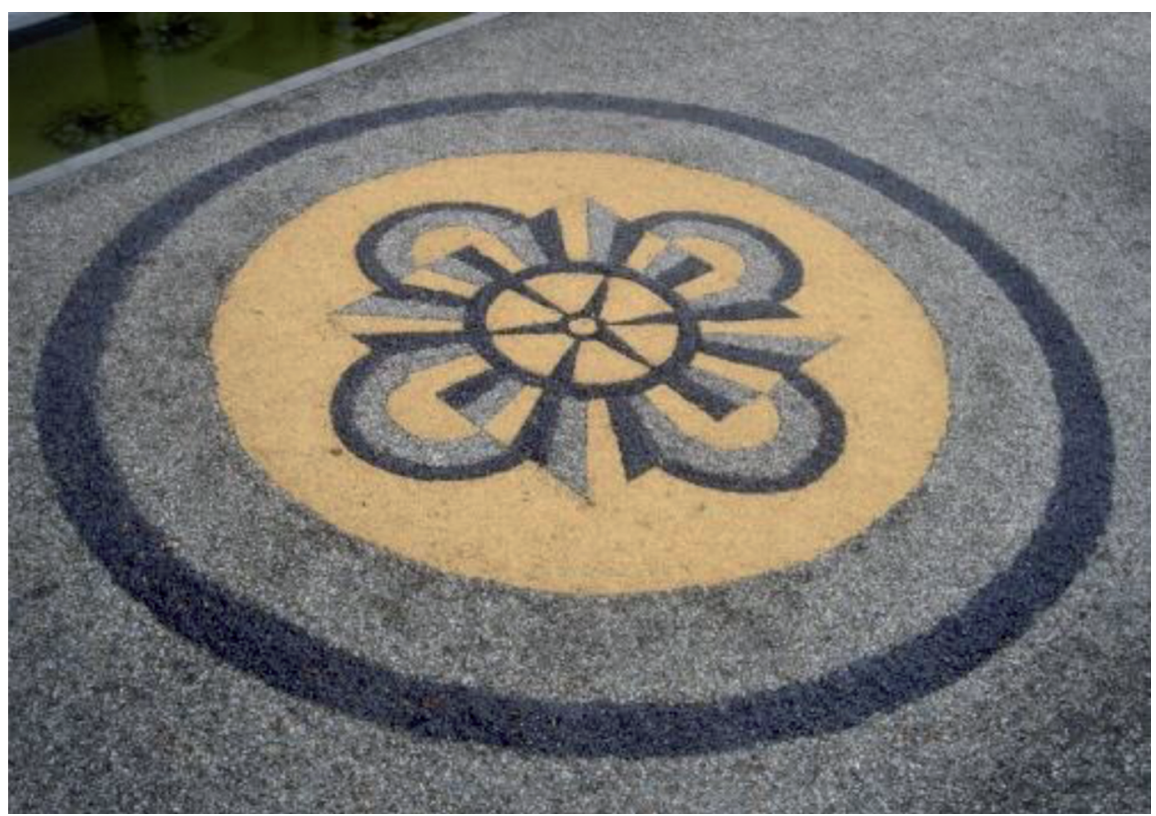

Abb. 1: Installation „jetzt geht es um die Wurst“ (Bernhard Huwiler, 2006)

72 Im Folgenden wird die alltagssprachliche Verwendung mit „Mandala“ - ohne diakritische Zeichen - angezeigt.

73 Vgl. http://www.ovra-archives.com/eMP/eMuseumPlus?service=ExternalInterface\&module= artist\&objectId=10000125\&viewType=detailView (20.02.2017). 


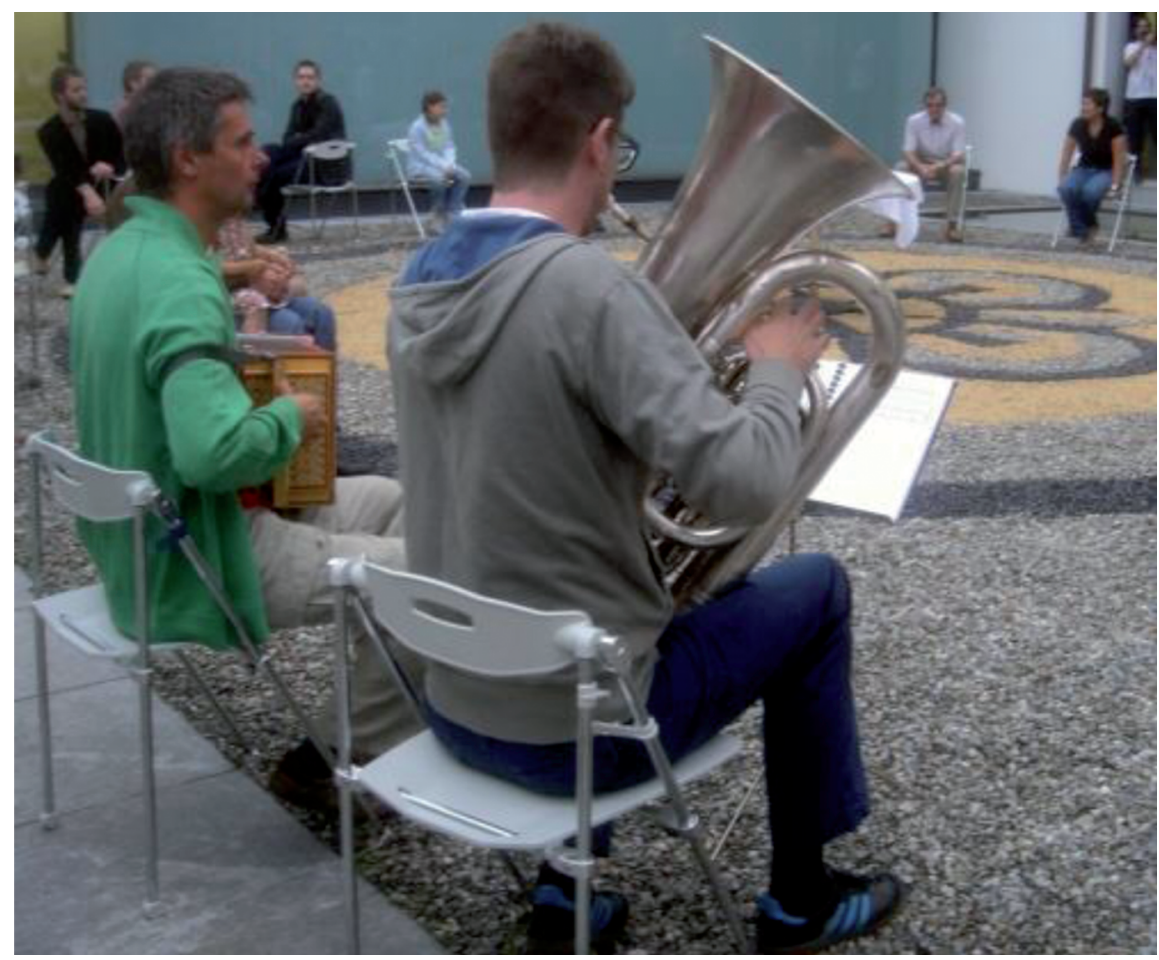

Abb. 2: Performance ,jetzt geht es um die Wurst“"vom 07.07.2006 (Abb. 1-3: @ Bernhard Huwiler)

In der besagten „Aktion“ hatte der Künstler aus unterschiedlich gefärbten Steinen eine Form geschaffen, die er selbst im alltagssprachlichen Sinn als „Mandala“ bezeichnete und die in gewissen Merkmalen, wie unten ausgeführt wird, einem Maṇḍala glich. Nach Aussage des Künstlers dachte dieser allerdings im Rahmen dieser Ausstellung nicht an eine religiöse Verwendung. Gewählt hatte er es vorrangig als ästhetisch ansprechende Form, die allerdings auch eine spirituelle Besinnung ermögliche. In der „Aktion“ definierte das Mandala eine Bühne von acht Metern Durchmesser, auf welchem die Performance ,jetzt geht es um die Wurst“ stattfand. ${ }^{74}$ In der Performance am Tag der Vernissage, in welchem Musiker zu dem bekannten Spiel „Reise nach Jerusalem“ aufspielten, wurde das

74 Bei diesem Spiel setzen sich die Mitspielenden beim Aussetzen der Musik auf die je um einen reduzierten Stühle. In diesem Fall wurde den Siegern eine große Wurst aufgetischt (dies bezog sich nach Aussage des Künstlers auf die Dreigroschenoper von Brecht, in der es heißt, dass zuerst das Fressen und dann erst die Moral komme). 
Mandala betreten und zum Großteil verwischt. Dies bringt gut zum Ausdruck, dass religiöse Achtungserweise (als eine Form „deontischer Macht“) ursprünglich nicht intendiert waren. Das Mandala sollte seine Rolle in dieser Funktion erschöpfen; an eine Wiederherstellung der klaren Form war nicht gedacht.

Bei genauerer Betrachtung der Installation erkennt man, dass der innere Bereich aus sechs gleichfarbigen Sektoren und einem punktförmigen Mittelpunkt bestand, von dem aus strahlenförmige Verbindungen zu einem Kreis aufschlossen. Diese kreisförmige innere Sektion wurde von einem quadratischen Feld umschlossen, welches wiederum nach außen weisende Strahlen aufwies. Beides entspricht meines Wissens keiner geläufigen buddhistischen Ikonologie. Der Künstler hatte sich hier denn auch vielmehr an dem auf den katholischen Heiligen „Bruder Klaus“, Nikolas von der Flüe (1417-1487), zurückgehenden sechsspeichigen „Sachsler Meditationsbild“75 orientiert, welches im Innern des Mandala Ausdruck fand, ohne dass diesem allerdings besondere ikonische Dignität verliehen wurde. Dieses Meditationsbild aus dem 15. Jahrhundert versinnbildlicht sechs Werke der Barmherzigkeit (Kranke besuchen, Fremde beherbergen usw.). Die vier Tore des Mandala und der die zentrale Struktur umgebende Kreis zeigen hingegen deutliche formale, wenn auch nicht farbliche Anklänge etwa zum Kālacakra-Maṇdala. ${ }^{76}$ Bevor die reguläre Ausstellung anlief, erschien ein Artikel von Otto Odermatt aus Seelisberg, ${ }^{77}$ der dazu aufrief, das Mandala nicht zu zerstören. ${ }^{78}$ In seinem Artikel führte Odermatt aus, dass ein Mandala den „göttlichen Ursprung der gesamten Schöpfung“ darstelle, „den jeder Mensch auch in sich hat, und den er durch die Meditation in sich erfahren“ könne. Es in einem „darwinistischen Überlebenskampf“ - der in der Performance „jetzt geht es um die Wurst“ nach der Intention von Huwiler umgesetzt wurde - zu zerstören,

75 Vgl. http://www.nvf.ch/rad1.asp (10.03.2017). In der Pfarrkirche von Sachseln (Innerschweiz) befindet sich eine originalgetreue Kopie der Betrachtungstafel, welche um 1475/80 gemalt und dem Eremiten geschenkt wurde. Hauptbestandteil des Bildes sind die beiden Kreise in der Mitte: ein innerer mit dem Gottesantlitz sowie ein äußerer, der durch je drei aus- und einlaufende Strahlen mit dem Inneren verbunden ist. Den Strahlen sind sechs Medaillons zugeordnet, die jeweils ein Werk der Barmherzigkeit präsentieren. Bei der quadratischen Form ließ sich der Künstler vom Schloss Rudenz am Urnersee inspirieren. Die Farben der Steine des Mandala wiederum beziehen sich auf die Farben des Kantonswappens von Uri (gelb und schwarz) (Mitteilungen des Künstlers, 03.04.2017).

76 Vgl. z.B. das 2007 aus Sand gefertigte Kālacakra-Maṇḍala, das Mönche des Namgyal Klosters, Dharamsala, Indien, bzw. Ithaca, USA, im Johnson Museum (Ithaca, New York), anfertigten (siehe http://museum.cornell.edu/exhibitions/tibetan-buddhist-mandalas\#\&gid=1\&pid=3, 01.03.2017). 77 Otto Odermatt ist unter anderem Leiter des Zentrum für „Transzendentale Meditation“, Seelisberg (vgl. http://schweiz.tm.org/web/seelisberg/kontakt, 01.03.2017).

78 Vgl. Neue Urner Zeitung, 25.04.2006. 
widerspreche sowohl der Intention des Meditationsbildes von Bruder Klaus wie auch dem buddhistischen Verständnis, in welchem mit der rituellen Auflösung und anschließenden Zerstreuung des Mandala „Buddhisten ihre Umgebung und die ganze Welt [segnen]“. ${ }^{79}$ Hintergrund der buddhistischen Auffassung, die hier nicht vertieft dargestellt werden kann, ist, dass ein Maṇdala als ein Abbild der kosmologischen Ordnung und seiner spirituellen Kräfte erachtet wird, in dessen Zentrum vorübergehend eine Gottheit (im Rang eines Buddhas) Wohnsitz nimmt (und weitere Gottheiten an anderen Orten desselben). ${ }^{80}$

Für den Künstler jedenfalls leitete diese Äußerung eine Wende ein gegenüber dem, was er geschaffen hatte. ${ }^{81}$ Der Vorwurf des blasphemischen Umgangs mit dem „heiligen Bild“ (Odermatt) beschäftigte ihn intensiv, so dass er sich schlussendlich entschied, das Mandala physisch zu rekonstruieren und rituell auflösen zu lassen. So suchte er, in Abstimmung und mit Unterstützung der Ausstellungsleitenden, das Klösterliche Tibet-Institut in Rikon (Kanton Zürich) ${ }^{82}$ auf, um die dort lebenden tibetischen Mönche zu bitten, das Mandala (bzw., jetzt angemessener, das Maṇdala) in einem Abschlussritual auszuweihen. Diesem Wunsch entsprachen die Mönche gerne, und dies, wie der Künstler anmerkt, mit professioneller Ernsthaftigkeit. So erkundigte sich ein Mönch, welcher Gottheit das Maṇ̣ala denn geweiht sei. Der Künstler erklärte, offenbar hinreichend zur Durchführung des Rituals, dass das Mạ̣dala der „Barmherzigkeit“ gewidmet sei.

Zur Finissage reiste eine Delegation von fünf buddhistischen Mönchen aus Rikon an, um das Auflösungsritual zu vollziehen (siehe Abb. 3). Die Ausweihung des Maṇdala geschah durch gemeinsame Rezitation und Gebet. Anschließend

79 Zitiert nach dem Leserbrief Odermatts (Neue Urner Zeitung; mit freundlichem Dank an Otto Odermatt für die Übersendung).

80 Ein buddhistisches Mandala (Tibetisch dkyil 'khor) kann als ein die kosmische Weltordnung repräsentierendes, symmetrisches Diagramm definiert werden, welches im inneren Kreis aus Sektoren besteht, die von umfassenderen Quadraten und wiederum umfassenderen Kreisen umschlossen sind. Es dient vorrangig dem Zweck einer kollektiven ritualisierten Meditation, in der bestimmte Gottheiten visualisiert werden. In einem gemeinschaftlichen Ritual bewegen sich die Praktizierenden in meditativer Versenkung zu dieser zentralen Gottheit hin und identifizieren oder vereinigen sich schließlich mit derselben. Entsprechend normativer Texte zur Beschreibung des Rituals dient das Maṇḍala der Vergegenwärtigung aller seiner Aspekte als Teil der Manifestation des ganzen Kosmos, welches auf das Erwachen des historischen Buddha bzw. generell eines Buddha hinweise (Sanskrit bodhi-mandala). Vgl. den guten Überblick zur komplexen Symbolik des Mandala in Martin Brauen, Das Mandala. Der heilige Kreis im tantrischen Buddhismus (DuMont: Köln, 1992).

81 Gespräch vom 03.03.2017, Bern.

82 Gegründet 1968 als religiöses Zentrum für die in die Schweiz migrierten Tibeter; vgl. Jens Schlieter, Marietta Kind und Tina Lauer, Die zweite Generation der Tibeter in der Schweiz: Identitätsaushandlungen und Formen buddhistischer Religiosität (Seismo: Zürich, 2014), 57-84. 


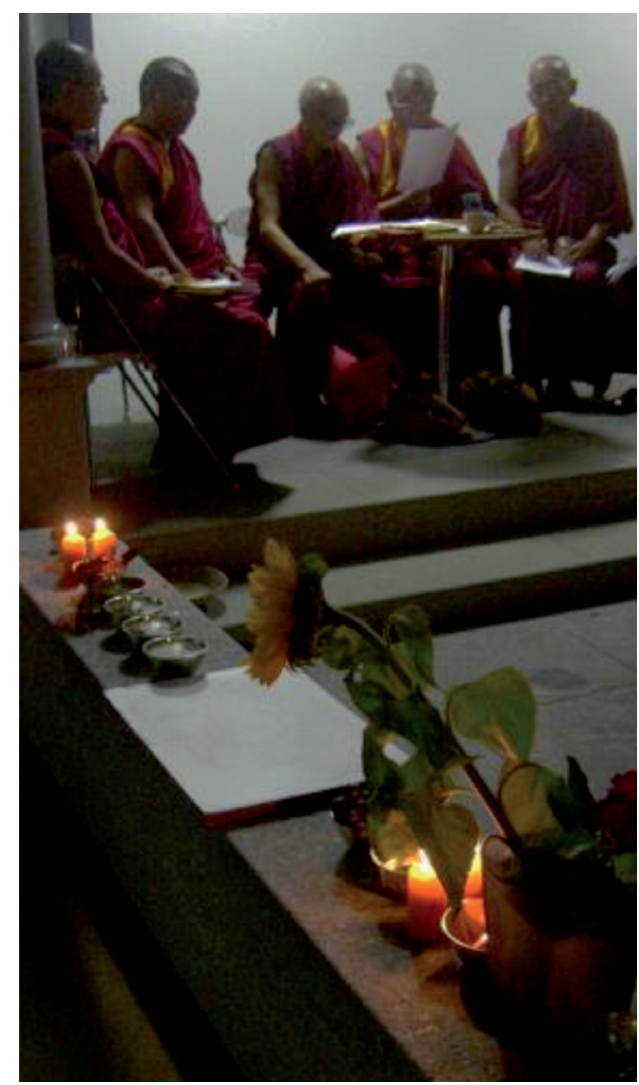

Abb. 3: Ritual zur Ausweihung des Mạ̣ḍala durch Mönche des klösterlichen Tibet-Instituts Rikon (Schweiz)

löste es ein Mönch mit Stockstrichen rituell auf, woraufhin es zusammengestrichen und physisch aufgelöst werden konnte.

Oben wurde argumentiert, dass etwas dann zu einem Symbol werden kann, wenn es durch eine Deutungsgemeinschaft in kollektiver Intentionalität als soziale Tatsache konstituiert und anerkannt wird, während für eine deskriptive Perspektive eingefordert werden muss, zugleich auch die Herstellungsabsicht bzw. die Referentialität mit nachzuvollziehen. Auf dieses Beispiel aus der realen Welt angewendet, fällt ins Auge, dass es zunächst eine Einzelperson war (und übrigens kein Vertreter einer buddhistischen Gemeinschaft), die auf den Charakter des Mandala als eines sakralen Symbols aufmerksam gemacht hatte. Die tibetischen Buddhisten behandelten es - trotz seiner ikonographischen Abweichungen und der ungewohnten Widmung - ab dem Punkt als Maṇḍla, als sie dazu eingeladen wurden (Transport und Bewirtung eingeschlossen), es rituell auszuweihen. 
So bleiben zwei, und vielleicht sogar drei konkurrierende Deutungen: Nach der ursprünglichen Absicht des Künstlers und seiner Deutungsgemeinschaft (die Kuratoren, Besucher der Kunstausstellung usw.) war es zunächst ein Kunstwerk und kein religiöses Symbol; nach der religiösen Deutung ist es trotz seiner atypischen Ikonologie und dystopischen Lokalität - einer Kunstausstellung - ein vollwertiges Maṇdala und nicht lediglich ein Kunstwerk. ${ }^{83}$ Nach einer (möglicherweise durch die tibetischen Mönche vertretenen) dritten Position ist es ab dann ein Maṇdala, wenn andere das Bedürfnis äußern, es möge als ein solches betrachtet werden.

Zwar kann an der obigen Bestimmung festgehalten werden, dass ein ikonisches Symbol nur dann (und nur solange) eine Bedeutung erhält, wenn (und wie) es durch eine Deutungsregel in Form einer Aussage ersetzt werden kann und über die Zulässigkeit der Ersetzung in einer Sprachgemeinschaft weitgehend Einigkeit herrscht. Wie verhält es sich aber mit dem geforderten Nachvollzug der Referentialität, die bei der Erschaffung des Objekts maßgeblich war? Und schafft die Tatsache, dass sich die Ausstellenden dazu entschlossen, ein buddhistisches Ausweihungsritual zu erbitten, der Deutung, dass es (von vorneherein) ein buddhistisches Symbol war, post festum Legitimität?

Um zunächst noch einmal zu Searle zurückzukehren, wird an dem Beispiel sehr gut deutlich, dass es nicht das „ikonische Etwas“ ist, welches eine Symbolwirkung erzeugt. Wäre der Blasphemie-Vorwurf nicht erhoben worden, wäre das Mandala nicht zum Maṇdala mutiert bzw. - im emischen Diskurs - transsubstantiiert. Erst in religiös motivierter, verbal oder schriftlich artikulierter Anschlusskommunikation wird eine transzendente Bedeutung in dem nun zum ikonischen Symbol erhobenen konkreten Objekt verankert, die in Folge erlaubt, das Objekt (ex post, aber zugleich immer schon) als Symbol zu sehen. Die Erzeugung der Symbolwirkung kann, wie gesagt, nur mit Referenz auf die kollektive Intentionalität spezifischer Deutungsgemeinschaften erklärt werden. Werden nun deklarative Sprechakte vollzogen, in und mit denen es zum buddhistischen Maṇulala - und somit zu einer institutionellen Tatsache - wird, so kommt ihm, in den Worten Searles, eine „deontische Macht“ zu. „Deontisch“ besagt hier, dass „sein-sollende“ Qualitäten erzeugt werden, also öffentliche Festlegungen von

83 Nur nebenbei sei angemerkt, dass Searles Auffassung, die institutionelle Wirklichkeit sei zwar ontologisch subjektiv, aber dennoch epistemisch objektiv beurteilbar, auf Widerspruch gestoßen ist (vgl. die Diskussion in Rota, „Religion”: 428-430). Searles Aussagen beziehen sich auf die Ebene der Gesamtgesellschaft, die die Variabilität von „institutionelle Tatsachen“ innerhalb spezifischer Gemeinschaften über den „Kontext“ zu bestimmen sucht (X gilt als Y im Kontext K), wobei Searles Interesse sich vorrangig auf die Möglichkeit sozialer Tatsachen überhaupt richtet, und zum „Kontext“ hingegen nur wenig ausgeführt wird (vgl. Searle, Konstruktion, 52-59.126). 
Rechten, Pflichten, Befugnissen, Erfordernissen, Beglaubigungen oder Genehmigungen. ${ }^{84}$ Searle macht an zahlreichen Beispielen deutlich, dass es freilich die Sprache - genauer: die Anerkennung öffentlicher Sprechakte - ist, welche diese Phänomene von Verpflichtungen, Verantwortungen und Rechten überhaupt erst schafft. ${ }^{85}$

An dem Beispiel wird nun allerdings auch offenbar, dass sich die Deutungsgemeinschaft, in der das Mandala als Maṇdala verstanden wird, nicht auf den Nachvollzug der Intention des Künstlers beruft, ja, diese Intention als nicht für entscheidend erachtet. ${ }^{86}$ Meines Erachtens ist davon auszugehen, dass religiöse Deutungsgemeinschaften dazu tendieren, die Erzeugungsakte von dem, was ex post als ein religiöses Symbol erkannt wird, auszublenden - ein Vorgang, den Blumenberg, wie oben angemerkt, noch dem „Symbol“ zuschreibt, nämlich die Beobachtung, dass es sich gegen seine eigene Herkunft verschließe. Aus der Außenperspektive bleibt aber das Faktum in Kraft, dass dem Mandala als künstlerischem, ikonischem Objekt entsprochen wird, indem die Intention seiner Erschaffung mitvollzogen wird. Aus der Anerkennung der buddhistischen Interpretation, dass es sich um ein Maṇdala handele, die sich augenfällig in der Zustimmung zum Auflösungsritual ausdrückt, folgt allerdings nicht, dass es sich auch außerhalb der buddhistischen Deutungsgemeinschaft um die „religiös-institutionelle Tatsache“ eines Mandala handele. Es ist nicht die Anerkennung des ikonischen Symbols als solchem aus dem rituellen Umgang anderer abzuleiten, sondern es bezeugt vielmehr die Achtung für eine spezifische Deutungsgemeinschaft und ihre religiösen Gefühle und Überzeugungen, die sich auf den Umgang mit dem Objekt beziehen.

84 Vgl. John R. Searle, Wie wir die soziale Welt machen: Die Struktur der menschlichen Zivilisation (Frankfurt a.M.: Suhrkamp, 2012), 208.

85 Vgl. ebd., 145.

86 Mögliche Begründungen könnten z.B. etwa ein Maṇdala im Sinne Carl Gustav Jungs als universelles Symbol des kollektiven Unbewussten verstehen und, solchen Perspektiven eine buddhistische Wendung gebend, davon ausgehen, dass der Kunstschaffende sich unbewusst buddhistisch artikuliert habe - vgl. Ders., Mandala. Bilder aus dem Unbewussten (Olten: Walter Verlag, 1977). 


\section{6 „Ein religiöses Symbol im Straßendreck“: Der Buddha vom Viktualienmarkt}

Um diesen entscheidenden Punkt weiter zu verdeutlichen, lässt sich ein weiteres signifikantes Fallbeispiel einer öffentlichen Kunstinstallation heranziehen: „Made in Dresden“, geschaffen von dem malaysisch-britischen Künstler Han Chong im Rahmen der vom Kulturreferat München veranstalteten Aktion „A Space Called Public - hoffentlich öffentlich“ (2013). Die Installation bestand aus der Skulptur eines ca. dreieinhalb Meter hohen, goldfarbenen Buddha, der in klassischer Ikonographie meditierend im Lotossitz gefertigt wurde, in der Installation allerdings auf dem Rücken lag, mit leichter vertikaler Drehung und etwas tiefer liegendem Kopf, so dass die auf der voll abschließenden Unterseite der Skulptur angebrachte Inschrift „Made in Dresden“ für die Betrachtenden gut exponiert gelesen werden konnte (siehe Abb. 4).

In der Beschreibung des Münchner Kulturreferats hieß es über die Installation und die Intention des Künstlers, dass der Buddha einen „überdimensionalen Souvenirartikel“ darstelle, der darauf aufmerksam mache, dass die Figur „zu >einem Dekorationsartikel fern des spirituellen Kontexts geworden“ sei und sich

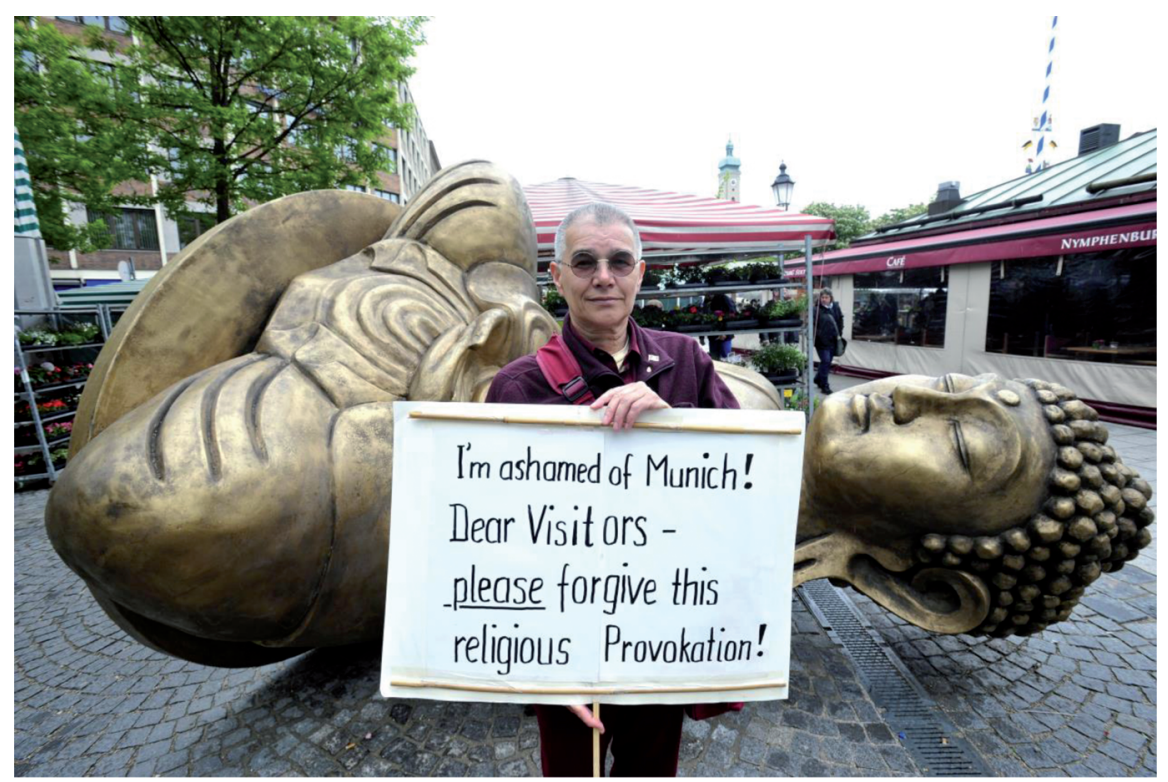

Abb. 4: „Die deutsche buddhistische Nonne Tenzin Wangmo protestiert“, († Markus Schlaf (tz online, 18.06.2013) 
anlässlich der Installation „die Frage nach Authentizität“ stelle. „Made in Dresden“ verweise darauf, dass Asiatica heute global hergestellt würden. ${ }^{87}$ Eine religiöse Bedeutung der Installation ist seitens des Künstlers - jedenfalls nach den begleitenden Texten des Programmhefts als auch den auf die Proteste folgenden Stellungnahmen - nicht intendiert gewesen. Trotzdem formierte sich schnell ein Protest, der sich in einer erstaunlichen Vehemenz äußerte. Stellvertretend sei dazu aus dem Briefwechsel zitiert, den die „Deutsche Buddhistische Union“ an den Münchner Kulturreferenten Hans-Georg Küppers adressierte. In einem ersten Brief wurde darauf hingewiesen, dass „eine derartige Zurschaustellung eines religiösen Symbols in der Öffentlichkeit“ bei Buddhisten „als Beleidigung und Herabwürdigung ihrer religiösen Überzeugungen empfunden werden“ müsse. ${ }^{88}$ In seiner Antwort bekräftigte Küppers, der „buddhistische Künstler“ habe nach eigener Auskunft „keinesfalls eine religiöse Provokation“ im Sinn gehabt. Die spezielle Lage sei nötig gewesen, um die an der Unterseite befindliche Inschrift sichtbar zu exponieren. ${ }^{89}$ Die DBU wiederum verschärfte in einer Rückantwort den Ton und ließ verlauten, dass die „Freiheit der Kunst“ nichts daran ändere, dass, aus öffentlichen Mitteln finanziert, ,auf dem Viktualienmarkt unübersehbar ein religiöses Symbol im Straßendreck liegt. Ob dieses Symbol nun in Asien oder in Dresden, von einem nepalesischen Kunsthandwerker oder von Herrn Han Chong hergestellt wurde, ist dabei völlig irrelevant“, und es sei „die Respektlosigkeit und die fehlende Sensibilität im Umgang mit einem religiösen Symbol, das in diesem Fall auch noch das Abbild eines von Millionen Menschen verehrten Religionsstifters ist“, die im Mittelpunkt der Kritik stehe. ${ }^{90}$ Zuletzt erklärte eine vom damaligen Münchner Oberbürgermeister Christian Ude unterzeichnete briefliche Antwort an die Thailändische Honorargeneralkonsulin, die „liegende Buddhaskulptur“ sei „kein Ritual- oder Kultgegenstand, sondern Han Chongs kritischer Kommentar zur beliebigen Verwendung eines religiösen Symbols“. ${ }^{91}$ Trotz weiterer Protestaktionen, die bis zu einer Demonstration thailändischer Buddhisten vor der Deutschen Botschaft in Bangkok reichten, ${ }^{92}$ verblieb der Buddha über die geplante Dauer auf dem Viktualienmarkt. Im Anschluss an die Ausstellung wurde die Skulptur, wohl nicht zuletzt durch die

87 Begleittext zur Ausstellung, http://www.aspacecalledpublic.de/ascp4.html\#han_chong, 01.03.2017; Abb. der Skulptur in ihrem Setting ebendort.

88 Brief vom 21.05.2013. Dieser und die im Folgenden zitierten Dokumente sind auf folgender Seite abrufbar: https://unbuddhist.com/2013/07/05/die-glosse-06/\#more-2502 (10.03.2017).

89 Brief vom 23.05.2013.

90 Brief vom 29.05.2013 (unterzeichnet von Sogen Ralf Boeck).

91 Brief vom 24.06.2013.

92 “Proteste in Thailand wegen Buddha am Viktualienmarkt," dpa (02.07.2013). 
Proteste befördert, dem Bodhicharya-Zentrum in Berlin geschenkt, welchem sie nun als zentrale Buddhastatue ihrer Meditationshalle dient. ${ }^{93}$ Von Seiten des aufnehmenden Zentrums hieß es übrigens, man müsse den Unterschied beachten zwischen einem „Kunstwerk“ und „einer Buddha-Statue, die für religiöse Zwecke gefertigt wurde. Von daher sehen wir die kritischen Reaktionen auf die Installation einiger Buddhisten kritisch“. ${ }^{94}$ Diese emische Stellungnahme erachtet also, wie oben für die Außensicht gefordert, die Intention des Produzierenden als zentral.

Nun könnte angesichts dieses Beispiels eine Revision des oben Gesagten gefordert werden: Im Falle einer der klassischen Ikonologie folgenden Darstellung eines Buddha sei auch in externer Perspektive fraglos gültig, dass es sich um ein religiöses Symbol handele. In einem mehrheitlich buddhistisch geprägten Land wie z.B. Thailand mag sicherlich zutreffen, dass sich einzelne dem Wissen, in einer solchen Skulptur als religiöse Repräsentation des Buddha (bzw. in ihr die Präsenz des Buddha) zu sehen, d.h. der Verehrung als sozial institutionalisierter Tatsache, nur schwer entziehen können. So argumentierten übrigens auch viele, die im wahrsten Sinne des Wortes eine „Besserstellung“ des Buddhas in München forderten - nämlich mit Verweis auf Besucher aus buddhistischen Ländern (vgl. Abb. 4). In der westlichen Spätmoderne, in der Buddhas in der Tat allerorten zu dekorativen Zwecken ausgestellt werden, ist es allerdings nicht ohne weiteres möglich zu entscheiden, in welchem situativen Kontext einer Buddhastatue im religiösen Sinne deontische Macht zukommen soll, und in welchem nicht. $\mathrm{Zu}$ erinnern ist hier nur an die in vielen Gartencentern käuflich zu erwerbenden, massenproduzierten Buddhastatuen, die nach Wissen des Verfassers bislang keine größere Protestaktion auslösten.

So wurde wiederum durch die Kritiker der Installation darauf verwiesen, dass der „entstellte“ Buddha im öffentlichen Raum zu sehen sei - und dies die eigentliche Provokation sei. Nach den Aussagen des Künstlers und der Veranstalter zu urteilen (und diese müssen für uns gelten, solange keine Gegenteiliges behauptende Quellen auftauchen), war eine religiöse Bedeutung nicht intendiert, und damit ist auch keine „böswillige Provokation“ im Sinne der europäischen Rechtsprechung (vgl. oben) gegeben. Dennoch ist sich der Künstler, wie dem obigen Zitat aus dem Programmheft (ein „Dekorationsartikel fern des spirituellen Kontexts“) zu entnehmen ist, offenbar der möglichen religiösen

93 Das Bodhicharya-Zentrum in Berlin, unter der spirituellen Leitung von Ringu Tulku Rinpoche (Sikkim, Indien), gehört der Linie der Karma Kagyü des tibetischen Buddhismus an (spirituelle Schirmherrschaft: Ogyen Trinley Dorje) und vertritt die „nicht-sektarische“ (Rime) Richtung.

94 Brief vom 10.10.2013, mit freundlichem Dank an Tenzin Peljor (Residenzmönch, Bodhicharya Berlin) für die Überlassung des Materials. 
Bedeutungszuschreibungen der Statue bewusst gewesen, spielt er doch explizit mit deren Differenz zur Vermarktung und Ästhetisierung zahlloser Buddhas in der globalen Moderne.

Auch dieses Beispiel macht augenfällig, dass die Rekonstruktion der Bedeutung eines religiösen Symbols aus der Ähnlichkeitsbeziehung zum Symbolisierten im Theoriekontext nicht tragfähig ist. Fraglos steht aber in diesem Fall die Statue in einer Ähnlichkeitsbeziehung zu anderen Statuen, die ihrerseits religiös verwendet werden. An die letztere Beziehung, so meine These, schließt die artikulierte Empörung an, wenn es um den sakralen Status derselben im profanierten öffentlichen Raum geht. In theoretischer Rekonstruktion besteht aber zwischen der zweiten, konkreten Ähnlichkeit und der ersteren, die von einem „Symbolisierten“ ausgeht, ein entscheidender Unterschied. Zudem wird erneut sichtbar, dass die Herstellungsabsicht aus emischer Sicht oft gänzlich außer Acht gelassen wird. Was gilt, ist der gekippte Buddha im Straßenstaub. Die Intention der Kunstschaffenden wird interessanterweise anscheinend nur dann einbezogen, wenn sich der Verdacht regt, dass eine Provokation beabsichtigt war. Überdies ist zu bemerken, dass es, ebenso wie im Fall des Mandala, schriftliche Appelle waren, die mindestens implizit den ja auch juristisch relevanten Vorwurf der Blasphemie artikulierten. Vor allem im zweiten Fallbeispiel, in welchem seitens der Ausstellenden kein Entgegenkommen gezeigt wurde, können die Briefe der DBU als wichtiger Faktor in der sich entwickelnden Dynamik, die zur Mobilisierung und Eskalation führte, angesprochen werden. Es sind also wiederum vor allem sprachlich-kommunikative Reaktionen auf die Installationen, denen zentrale Bedeutung zukommt.

Im Verlauf der Ausstellung wurden nun dem Buddha, wie Fotografien und Augenzeugen bestätigen, zunehmend Opfergaben (Kerzen, Blumen, Wasserschalen usw.) dargebracht.

$\mathrm{Zu}$ guter Letzt kam es sogar zu einer „Protest-Meditation“, bei der etwa 30 Buddhisten vor der Installation zusätzlich einen goldenen Buddha unter einem Pavillon platzierten und sich so auf beide Skulpturen ausrichteten (siehe Abb. 5). Hier wird die Frage noch dringlicher, wie mit der in kollektiver Intentionalität als Verehrungspraxis deutlichen Erschaffung eines religiösen Symbols umgegangen werden soll. Besonders bemerkenswert ist in diesem Beispiel, dass wiederum das Kunstobjekt post festum als religiöses Symbol anerkannt wurde, indem der Künstler wie die Ausstellungsleitenden der Schenkung und Überführung des Buddha an ein buddhistisches Zentrum zustimmten. Bedeutet dies, dass auch die Kunstschaffenden durch die Augen anderer ihr Werk „religiös sehen“ lernten? Oder wollten sie sich dem Toleranzgebot letztlich nicht entziehen? Oder schätzten sie die religiöse Deutung als eine Bezeugung höchsten Respekts, die ihrer Kunst gleichsam eine andere Form von Hochschätzung zukommen ließ? 


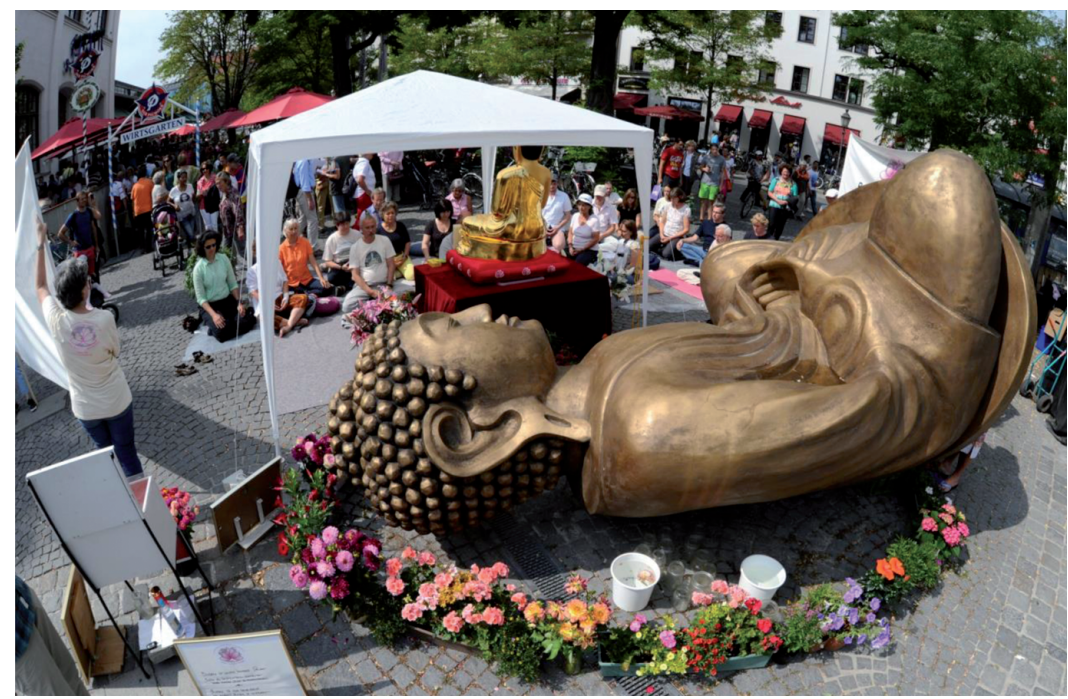

Abb. 5: „Protest-Meditation vor umgestürzter Buddhastatue in München“ (@ keystone; erschienen im Stern, 24.08.2013)

\section{Religiöse Symbole und die respektierende Anerkennung der kollektiven Intentionalität von Deutungsgemeinschaften}

Die obigen Argumente zusammenfassend, kann festgehalten werden, dass die Präsenz des Symbolisierten im Symbol als in kollektiver Intentionalität vermittelte, in das Symbol verlegte Erwartungen und Erwartungshaltungen erachtet werden kann. Damit geht einher, dass die relevanten anderen der eigenen Gemeinschaft auf genau dieselbe Weise dem Symbol gegenüber Respekt bezeugen, fehlende Achtungserweise hingegen bemerken und ggf. sanktionieren. In emischreligiöser Sicht wird hingegen von der Präsenz des Symbolisierten im ikonischen Symbol ausgegangen, welche über weitere diskursive, imaginative und rituelle Praktiken, wie auch der theoretischen Reflexion auf die selbigen, institutionell abgesichert wird. Im Sonderfall des öffentlichen Raumes werden auch von anderen Achtungserweise eingefordert, wobei interessanterweise einmal die Produktionsabsicht zählt (Blasphemie-Vorwurf) und ein anderes Mal nicht.

Eine Orientierung an der sinnlichen Wahrnehmung, die sich über das Faktum der beobachtungsrelativen, ontologisch subjektiven Basis mit Verweis auf die epistemische Objektivität des Symbolverstehens hinwegsetzen kann, führt hier 
nicht weiter. Eine solche Objektivität hätte überhaupt nur dann Bestand, wenn eine homogene Wahrnehmung über semantische Kommunikationsprozesse, in denen das, was gemeinsam an oder in etwas gesehen wird, abgesichert wird. Um dem Faktum der Institutionalisierung nicht nur des ikonischen Symbols, sondern darüber hinaus dessen sich auf „Ähnlichkeit“ berufender Genealogie sowie der kollektiven Repräsentation der in emischer Sicht von dem ikonischen Symbol ausgehenden religiösen Symbolwirkungen gerecht $\mathrm{zu}$ werden, möchte ich die Bezeichnung „effinktive Symbole“ vorschlagen. Aus dem Lateinischen ef-fingere (ex-, „aus“, und fingere „formen, bilden“), „nachformen, porträtieren“, abzuleiten, ${ }^{95}$ soll der Neologismus „Effinktion“ gleichermaßen „Effektivität“, „Funktion“ und „Fiktion“ anklingen lassen. Die „Effinktion“ ist effektiv, da vermittels kollektiver Intentionalität ein religiöses Symbol, welches überdies als selbsttätig wirkmächtig erachtet wird, institutionalisiert wird; sie erfüllt für religiöse Gemeinschaften eine zentrale „Funktion“, und sie trägt „fiktive“ Züge, da die Ähnlichkeit zu einer repräsentierten Transzendenz als im Symbol verankert angesehen, und ohne Referenz auf den Entstehungskontext gedeutet wird. Ein (in früherer Zeit oder anderen Kontexten religiöses) ikonisches Symbol, welches aber aktuell durch keine religiöse Deutungsgemeinschaft als Symbol in Anspruch genommen wird, wäre dann als „nicht effinktiv“ zu bezeichnen. Die „Effinktion“ eines religiösen Symbols ist allerdings nicht die agency des Dinges, sondern die Wirkmacht der in das Ding verlegten institutionellen Tatsache, die ihrerseits die normativen Erwartungen und kollektiven Repräsentationen der Deutungsgemeinschaft spiegelt. Tatsächlich kann sich diese Wirkmacht so eng an das zum Symbol erklärte Ding anlegen, dass deren Unterscheidung kaum mehr sichtbar ist.

Symbolon, im griechischen Kontext zunächst „die zwei Hälften einer Sache“, bezeichnet spätestens seit Aristophanes in Platons Symposion (191d) in mythischer Bedeutung die je eine Hälfte eines ursprünglichen Ganzen bzw. zwei zusammengehörige Kugelhälften (vgl. Aristoteles, Eudemische Ethik, 1239b). In dessen Konsequenz richtet sich bis heute das Interesse nur selten darauf, wie religiöse Symbole entstehen und wie es zu ihrer Akzeptanz als soziale Institutionen mit deontischer Macht kommt. Dennoch ist für den externen Beobachter die Differenz analytisch konstitutiv. Um eine Metapher zu verwenden, kann hier auf die „Magdeburger Halbkugeln“ verwiesen werden, mit denen Otto von Guericke 1654 demonstrierte, dass acht Pferde nicht in der Lage waren, zwei Halbkugeln zu trennen, denen mit einer Vakuumpumpe die Luft entzogen worden war. Auf

95 Vgl. Charlton T. Lewis, Charles Short, A Latin Dictionary (Oxford: The Clarendon Press, 1958), 630 (s.v.); vgl. effigies, „With the accessory idea of resemblance obtained by imitation, a likeness, portrait, image, effigy" (ebd.). 
vergleichbare Weise werden das ikonische Objekt und seine durch kollektive Intentionalität institutionalisierte Deutung aneinander gekoppelt. Sicherlich werden religiöse Gemeinschaften nicht ein beliebiges Objekt als „effinktives Symbol“ für sich reklamieren können, da die Plausibilität steigt, wenn eine Gemeinschaft auf sedimentierte Bedeutungen verweisen kann, die durch intensive analoge Verwendungen entstanden sind. Dennoch: Ein überzeitliches Passungsverhältnis, das sich, dem emischen Diskurs folgend, auf die Präsenz des Symbolisierten beruft und dessen sakrale Identifikation mit dem Objekt argumentativ über die Ähnlichkeit vornimmt, kommt aber, wie gesehen, genau dann in Bedrängnis, wenn konkurrierende Deutungsmodelle über das, was hier eigentlich vorliegt, existieren. Wie ist dann eine Entscheidung zu treffen, ob etwas ein religiöses - als im obigen Sinne „effinktives“ - Symbol sei oder nicht? Ontologisch ist dies jedenfalls nicht möglich, da die Ähnlichkeit allein (man denke nochmals an die verehrungsfreien Buddhas im Gartencenter) kein Urteil absichern kann. Ebensowenig ist der Kontext allein maßgeblich. Auch im öffentlichen Raum kann ein potentiell als Symbol zu lesendes „etwas“ unbemerkt bleiben. Aus religionswissenschaftlicher Sicht bleibt somit allein übrig, diese Frage nicht mit Referenz auf das als Symbol verstandene ikonische Objekt, sondern nur unter Bezug auf die spezifischen Wirkungen der deontischen Macht, die diesem in Deutungsgemeinschaften zugeschrieben werden, zu beantworten. Konkret heißt dies, die sich konkurrenzierenden Deutungen zu beschreiben und im jeweils konkreten Fall darzulegen, welche Erwartungshaltungen und Emotionen religiöse Deutungsgemeinschaften durch Sprechakte und andere kommunikative Wege einerseits, und Kunstschaffende, Ausstellende oder explizit säkulare Gemeinschaften andererseits äußern. Letztlich wird dies zur Ermessensfrage, wie die beteiligten Gemeinschaften im öffentlichen Raum ihren Deutungen durch rituelle Handlungen oder kollektiven Protest nachhaltig Ausdruck verleihen und was die eine wie auch die andere Partei verliert, wenn ihrer Deutung nicht stattgegeben wird. Diese Fragen sind natürlich letztlich keine religionswissenschaftlichen mehr, sondern politische. Kann eine provokative oder gar „bösartige“ Darstellungsabsicht ausgeschlossen werden, so bleibt es in der Entscheidung anderer (in den obigen Fällen der Kunstschaffenden und Ausstellungsorganisatoren und in anderen Fällen der Mehrheitsgesellschaft), dem Respekt vor den Emotionen und Deutungen religiöser Akteure einen höheren Stellenwert einzuräumen als der auch irritierende Reaktionen evozierenden - Dignität der Kunst.

Danksagung: Für zahlreiche hilfreiche Kommentare danke ich Karénina KollmarPaulenz, Volkhard Krech, Moritz Klenk und Michael Stausberg sowie Karolina Lisowski, Karin Mykytjuk, Andrea Rota und den Gutachtenden der ZfR. 